\title{
GENERAL VECTOR CALCULUS *
}

BY

\author{
JAMES BYRNIE SHAW
}

\section{INTRODUCTION}

This paper presents results from various papers read before the Society during a period of several years. These are indicated in the footnotes. The calculus is independent of the number of dimensions of the space in which the vectors are supposed to be placed. Indeed the vectors are for the most part supposed to be imbedded in a space of an infinity of dimensions, this infinity being denumerable sometimes but more often non-denumerable. In the sense in which the term is used every vector is an infinite vector as regards its dimensionality. The reader may always make the development concrete by thinking of a vector as a function of one or more variables, usually one, and involving a parameter whose values determine the "dimensionality" of the space. The values the parameter can assume constitute its "spectrum." It must be emphasized however that no one concrete representation is all that is meant, for the vector is in reality an abstract entity given by its definition, that is to say, postulationally. The case is analogous to that of "group" in which the "operators" are generally not operators at all, since they have nothing to operate upon, but are abstract entities, defined by postulates. Always to interpret vectors as directed line-segments or as expansions of functions is to limit the generality of the subject to no purpose, and actually to interfere with some of the processes. It is sufficient to notice that in any case the theorems may be tested out in any concrete representation. The author desires to state that he discriminates between the terms function, values of a function, expansion of a function. The function is the law which enables one to ascertain the values. The table of values is not the function. The expansion is the system of coefficients of the basis functions by means of which we may have a particular representation of the function. Further a system of coefficients does not constitute a vector; such a system the author calls a multiplex.

It is true that a multiplex appeals to many mathematicians as a sufficient entity to serve as vector, but a multiplex does not specify a vector till the basis (or in geo"metry the set of axes) is specified. A system of coefficients does not define the same function when attached to powers of $x$ as when attached to sines in a Fourier series; the vector $(1,2,3)$ has no meaning till the directions for the

* Presented to the Society at various meetings, 1909-1921.

Trans. Am. Math. Soc. 14. 
numbers are assigned; and $1-x^{2}$ is the same function whether expanded in a Fourier series or a Bessel series, though its multiplex is different. The essence indeed of a vector calculus lies in its ability to produce invariantive and covariantive expressions, and it does this because it uses vectors directly and not some particular representation of the vectors. A calculus which produces formulas in $x, y, z$, even though they may be at once replaced by $x^{\prime}, y^{\prime}, z^{\prime}$, and preserve the same formulas, may be an invariant calculus, but it is not a vector calculus. At the best, symbols which stand for such expressions referred to axes for their meaning are merely short-hand symbols.

The method developed here has been found by years of use to be flexible, smooth-running, simple, and to include all the systems of vectors now extant. Its expressions are all covariantive for the simple reason they have no dependence upon any system of reference. They are compact and readily interpreted in such applications as differential geometry. It furnishes the basis for integral equations, and the general theory of distributive operators. In the abstract the intention is to create a calculus of symbols which are defined by the rules of the calculus. Any entities that submit to these rules may be represented by the symbols so far as this calculus is concerned, though from other points of view they must be treated differently. These symbols stand for entities which are subject to certain processes. A process is not a function; it produces entities which are functions of the entities subject to the process, but the process is not the function. For instance we use a process called addition, and one called multiplication. There are several called " $A$ " processes which are not "multiplications." The author is aware that some mathematicians like to call some of these $A$ processes by such names as inner multiplication, outer multiplication, indeterminate multiplication, and the like, but he has not yet observed that this multiplicity of names has been any advantage in the development of the subject.

On the side of vectors in finite space (as to dimensions) this method includes the Clifford algebras, ${ }^{*}$ and might be supposed to be identical with the general treatment of Clifford. The question has been asked: Why not assume a system of units, $i_{1}, i_{2}, \cdots, i_{n}$, whose squares are -1 , and such that $i_{p} i_{q}=-i_{q} i_{p}$ when $p \neq q$ ? All the formulas in the finite cases may be worked out from this point of departure. They mint then be generalized to the case of a denumerable infinity of units. This would indeed at least simplify much of the extant work on the uses of orthogonal functions, vectors in a "Hilbert space," etc. But when we take up the most general case of a non-denumerable space it would fail. The present development has no reference to any dimensions.

* Consult Clifford's Works; Joly, Proce edings of the Royal Iris h A c a de m y (3), vol. 5 (1897), pp. 73-123; vol. 6 (1900), pp. 13-18; M'Aulay, P r o c e e d i n g s of the Royal Society of Edin burgh, vol. 28 (1908), pp. 503-585. 
This must be well understood by the reader, for it is fundamental. The Grassmann treatments are hampered by their assumption that the "outer product" of all the basis units of the system is a numerical value. Without knowing the number of dimensions of the space the formulas cannot always be written. The formulas here are equally valid whether the vectors lie in a space of three, three thousand, a denumerable number, or a non-denumerable number of dimensions. There is no basis, for every vector is an entity unique in itself. It defines with its numerical multiples a linear space, any two define with their numerical multiples and under addition a binary space, any $n$ an $n$-ary space,- - under certain restrictions to be noted.

\section{FUNDAMENTALS}

\section{Definitions}

1. Vectors. Vectors are represented in notation throughout by small Greek letters, or by expressions built up with small Greek letters, and other symbols. Capital Greek letters invariably mean operators. Roman (italic) letters mean numerical values selected from a given domain, or field. An exception sometimes is furnished in using $\pi$ in the ordinary sense.

Vectors are defined by stating the conditions to which they are subject. These are as follows.

(1) Each vector gives rise to a set of vectors, called its multiples, represented by writing the symbol of the vector and a symbol for a number (or mark) chosen from the given field, thus from $\alpha$ we have $a \alpha, 3 \alpha, \pi \alpha$, and the like. No distinction is made between $a \alpha$ and $\alpha a$. The multiples of a vector are as numerous as the marks or numbers of the field. Any one of the multiples may itself be taken as the initial vector and the others, including the original vector, are then multiples of it. No vector is a unit vector absolutely, but only relatively.

(2) In case we have one more vector which is not among the multiples of the vector $\alpha$, say $\beta$, then the multiples of $\alpha$ and the multiples of $\beta$ enable us to form a binary complex of vectors, indicated by $x \alpha+y \beta$, where $x$ and $y$ independently assume all values in the field. The vector $\xi=x \alpha+y \beta$ is a unique vector, but is said to be dependent upon $\alpha$ and $\beta$, or not to be linearly independent of them. We call the process of forming these vectors of the complex addition of vectors. We assume as a matter of definition that

$$
\xi=x \alpha+y \beta=y \beta+x \alpha,
$$

and further that, in the case of still a third vector, not in the complex of $\alpha$, and $\beta$, say $\gamma$, if $\xi=x \alpha+y \beta, \zeta=y \beta+z \gamma, \sigma=\xi+z \gamma, \tau=x \alpha+\zeta$, then in all cases $\sigma=\tau$.

This is called associativity of addition. 
(3) If $\xi=\sum x_{n} \alpha_{n}$, then the multiples of $\xi$, as $c \xi$, are also to be formed from the same multiples of $x_{1} \alpha_{1}, x_{2} \alpha_{2}, \cdots$, that is

$$
c \xi=\sum c x_{n} \alpha_{n} \text {. }
$$

We see that by this last condition

$$
x \alpha+y \alpha+\cdots=(x+y+\cdots) \alpha .
$$

(4) If 0 is included in the field, then we have vectors $0 \alpha, 0 \beta, \cdots$ and these are indistinguishable.

It follows that we may consider $\alpha+0 \xi=\alpha$ in all cases. Further we see that the multiples of $\alpha, \beta, \gamma$, etc., which determine a complex are to be considered as in the complex. Hence the vectors which determine a complex are not unique. Whatever vectors are used to determine a complex are called a basis for the complex in case they constitute an irreducible set, in the sense that no one of them is included in the complex of the others. That is the same as saying that $\alpha_{1}, \alpha_{2}, \cdots, \alpha_{n}$ form a basis for a complex in case

$$
0 \rho=x_{1} \alpha_{1}+x_{2} \alpha_{2}+\cdots+x_{n} \alpha_{n}
$$

only for $x_{1}=x_{2}=\cdots=x_{n}=0$. They are said then to be linearly independent.

(5) When the number of linearly independent vectors is not finite we indicate them by using a parameter attached to the vector symbol, sometimes as a subscript, as in the case of a denumerable infinity, thus $\alpha_{1}, \alpha_{2}, \cdots, \alpha_{\infty}$; and sometimes as a parameter in a parenthesis, as $\alpha(i)$, as in the case of a non-denumerable infinity. The complex in the first case is indicated by

$$
\xi=\sum_{1}^{\infty} x_{n} \alpha_{n} \quad \text { or } \quad \sum x_{n} \alpha(n) .
$$

The complex in the second case is indicated by

$$
\xi=\int_{a}^{b} x(i) \alpha(i) d i .
$$

As an instance of each case, so far as our conditions go we may consider that functions of a single variable are vectors. We would then have, using $z$ for the variable, $i$ for the parameter, in the first case, an instance from expansions in orthogonal functions,

$$
\xi(z)=\sum a_{n} \phi_{n}(z) ;
$$

in the second case, an instance from the expression of a function as a definite integral,

$$
\xi(z)=\int_{a}^{b} a(i) \phi(i, z) d i
$$


When we apply vectors to functions we need to note there are further restrictions on account of the problems of analysis. We are concerned here largely with the problems of algebra only.

When we undertake to find the coefficients (from the field) which would enable us to express a given vector as belonging to the complex of a given basis (finding the components of the vector), in case it is possible to express the vector thus, that is, in case the vector does belong to the complex, we say we resolve the given vector on the given basis. This is exemplified in the process of finding components of velocity, or force, or vector field, or Fourier coefficients, or solving a linear homogeneous integral equation. The conditions, in the function case again, that such resolution may take place, have been extensively studied in certain special cases. The study of such conditions as result from these analytic problems has been carried far by E. H. Moore, whose papers on General Analysis may be referred to. The definitions of norm, Hermitian square, generalized theorem of Pythagoras, etc., find their place in such investigations.

(6) Vectors are further subject to conditions which are the main object of the present paper. These result from the formation of vectors of grade 2, 3 , $\cdots$, and their combinations into complexes, and combinations into complexes of mixed grades. These will be treated separately under the heads: the accretive process, the decretive process, the Hamiltonian process. The latter will also be called the product.

\section{The ACCRETIVE PROCESS}

2. Alternants.* In general an expression is alternant when it changes sign with the interchange of two of its elements. The usage here is not contradictory to this but supplements it considerably. As an example of an alternant let us consider $n$ functions of $n$ different variables, giving us $n^{2}$ different functions

$$
\alpha_{i}\left(s_{j}\right) \quad(i, j=1,2, \cdots, n) .
$$

Then the expression $\sum \pm \alpha_{i_{1}}\left(s_{1}\right) \alpha_{i_{2}}\left(s_{2}\right) \cdots \alpha_{i_{n}}\left(s_{n}\right)$, where the subscripts $i$ are the numbers $1,2, \cdots, n$ in some permutation, all permutations occurring, and the sign is + or - according to the number of inversions, is an expression which changes sign if any two subscripts are interchanged. It is therefore an alternant.

The alternants we introduce are defined by the following conditions:

(1) From any $n$ vectors we may construct an alternant, written $A_{n} \cdot \alpha_{1} \alpha_{2}$ $\cdots \alpha_{n}$, called a simple vector of grade $n$.

* The remainder of part I contains the results of papers read before the Society as follows: Chicago Section, December 26, 1913, The two fundamental operations of general vector analysis; December 31, 1909, On Hamiltonian products; Southwestern Section, November 27, 1909, Scalars of lineal products. 
(2) The interchange of any two vectors changes the sign.

(3) If any one of the vectors is in a complex, say $\alpha_{i}=\sum a_{j} \beta_{j}$ where the $\sum$ may be used also to include the non-denumerable case

then

$$
\alpha(i)=\int a(j) \beta(j) d j,
$$

$$
A_{n} \cdot \alpha_{1} \alpha_{2} \cdots \alpha_{i} \cdots \alpha_{n}=\sum a_{j} A_{n} \cdot \alpha_{1} \alpha_{2} \cdots \beta_{j} \cdots \alpha_{n} .
$$

It follows at once that if any two vectors are equal the alternant vanishes, that if any vector is in the complex of the others the alternant vanishes, and that to any vector may be added any vector in the complex of the others without altering the alternant. The distributivity property (3) is very important.

(4) $A_{1} \cdot \alpha=\alpha$. The alternants begin with $A_{2}$. We preserve the notation since it is valid in general theorems.

(5) If $A_{n} \cdot \alpha_{1} \alpha_{2} \cdots \alpha_{n}=0$ then for some set of values $x_{1}, x_{2}, \cdots, x_{n}$ not all zero

$$
x_{1} \alpha_{1}+x_{2} \alpha_{2}+\cdots+x_{n} \alpha_{n}=0 .
$$

This makes either equation a necessary and sufficient condition for the other. It precludes using as the alternant defined above any alternant expression. For instance in quaternions we might use as $A_{2} \cdot \alpha \beta$ the expression $V \cdot \theta(\alpha) \theta(\beta)$ where $\theta(\mathrm{l}$ is a linear vector function, but it would be necessary to choose $\theta$ as non-singular. Otherwise the expression might vanish because $\alpha$ or $\beta$ happened to be an invariant line for the root 0 . Similarly we might take as $A_{2} \cdot \alpha \beta$ the expression

$$
\int_{a}^{b} \int_{a}^{t}[\alpha(s) \beta(t)-\beta(s) \alpha(t)] \phi(s) d s d t,
$$

but we should have to place restrictions upon what $\phi()$ could be. Indeed in either example we can consider that we do not really have the simple alternant, but a function of the alternant, for they may be written respectively $\Phi V \cdot \alpha \beta$, and $\Phi[\alpha(s) \beta(t)-\beta(s) \alpha(t)]$. The function might vanish when the elementary alternant does not. We limit the meaning of $A_{n}$ - otherwise, however, only by the postulates.

3 . Vector of grade $n .^{*}$ A basis of alternants of grade $n$ gives a complex of grade $n$, and any member of the complex is a vector of grade $n$. A vector of grade $n$ is indicated by $V_{n}$. It does not have to reduce to a single alternant, as for instance $A_{2} \cdot \alpha \beta+A_{2} \cdot \gamma \delta$ may or may not reduce to the form $A_{2} \cdot \epsilon \zeta$. Many vectors of grade $n$ do however reduce to single terms which are represented by the symbols $A_{n}$. Such is the case to be defined, which is written

\footnotetext{
*This is not the "polyadic" of some authors.
} 
$A_{n} \cdot \alpha_{1} \alpha_{2} \cdots \alpha_{n+k}$ where $k \neq 0$. Such expressions have properties to be proved which may be stated in terms of the permutation groups under which they are invariant. In case $k=0$ we might say that $A_{n} \cdot \alpha_{1} \alpha_{2} \cdots \alpha_{n}$ is invariant under the alternating group of permutations of its vectors, and changes sign for the remaining substitutions of the symmetric group. Other expressions constructed from an initial term by applying the substitutions of some group would be interesting and useful, but little study of them has occurred.

4. Accretive process. The accretive process is now defined by the equation $A_{p+q} \cdot A_{p} \cdot \alpha_{1} \alpha_{2} \cdots \alpha_{p} A_{q} \cdot \beta_{1} \beta_{2} \cdots \beta_{q}=A_{p+q} \cdot \alpha_{1} \alpha_{2} \cdots \alpha_{p} \beta_{1} \beta_{2} \cdots \beta_{q}$, where $p, q$, are any integers. Hence if the alternants are interchanged the sign is multiplied by $(-1)^{p q}$.

\section{The DECRETIVE PROCESS}

5. Scalars. The term scalar was first applied by Hamilton to quaternions which could be included in the ordinary number scale. It is used here in the same sense but with the further application to a process which gives numbers (marks) belonging to the field, from vectors, whatever the grade of the vectors. We shall use $A_{0}$ - to indicate such results. We define first:

(1) $A_{0} \cdot A_{n} \cdot \alpha_{1} \alpha_{2} \cdots \alpha_{n} A_{n} \cdot \beta_{1} \beta_{2} \cdots \beta_{n}$ is a number or mark of the coefficient field.

(2) $A_{0} \cdot A_{n} \alpha_{1} \alpha_{2} \cdots \alpha_{n} A_{n} \cdot \beta_{1} \beta_{2} \cdots \beta_{n}$

$$
=A_{0} \cdot A_{n} \cdot \beta_{1} \beta_{2} \cdots \beta_{n} A_{n} \cdot \alpha_{1} \alpha_{2} \cdots \alpha_{n} .
$$

This will be found to be a special case of a more general formula. In particular

$$
A_{0} \cdot \alpha \beta=A_{0} \cdot \beta \alpha \text {. }
$$

(3) $A_{0} \cdot A_{p}() A_{q}()=0, \quad p \neq q$.

(4) $A_{0} \cdot(P+Q+\cdots)(R+S+\cdots)$

$$
=A_{0} \cdot P R+A_{0} \cdot P S+\cdots+A_{0} \cdot Q R+A_{0} \cdot Q S+\cdots
$$

for any expressions $P, Q, \cdots, R, S, \cdots$. That is, $A_{0}$ is distributive.

Instances of the scalar process are found in the expression called inner product by some mathematicians. In geometric vectors it is usually defined to be the product of the lengths of the vectors by the cosine of their angle, which angle is defined for the space involved. In functions we have

$$
A_{0} \cdot \alpha \beta=\int_{a}^{b} \alpha(s) \beta(s) d s .
$$

We do not identify the scalar process $A_{0}$ with the scalar process in quaternions, though the latter is included. For we might take $A_{0} \cdot \alpha \beta=S \alpha \theta(\beta)$ where 
$\theta$ () is a self-transverse (symmetric, self-conjugate) linear vector operator which has no zero roots. Such a form is implied in the integration with a matrix defined by E. H. Moore in his General Analysis.

Scalars of vectors of grades higher than one are expressible by scalars of vectors of grade one.

6. Orthogonality. If the scalar of two vectors of any grade vanishes the two vectors are said to be orthogonal. A system of vectors may be mutually orthogonal, as for instance a system of mutually perpendicular vectors in space, or a system of functions such as $\alpha_{i}=\sqrt{2} \sin i \pi s$, under the definition

$$
A_{0} \cdot \alpha_{i} \alpha_{j}=\int_{0}^{1} \alpha_{i}(s) \alpha_{j}(s) d s
$$

Biorthogonality. Two systems of vectors $\alpha(i), \beta(j)$ are mutually biorthogonal in case we have

$$
\begin{aligned}
& A_{0} \cdot \alpha(i) \beta(j)=0, \quad i \neq j, \\
& A_{0} \cdot \alpha(i) \beta(i) \neq 0 .
\end{aligned}
$$

Biorthogonality and orthogonality play an important part in the problem of expansions mentioned earlier. For instance they enable us to find the coefficients under proper restrictions.

7. Decretive process. We are now in a position to define the general decretive process. We define first the expression

$$
A_{n-1} \cdot \alpha A_{n} \cdot \beta_{1} \beta_{2} \cdots \beta_{n}=\sum(-1)^{i-1} A_{0} \cdot \alpha \beta_{i} \cdot A_{n-1} \cdot \beta_{1} \cdots \beta_{n}
$$

where the $i$ written under $\beta_{1} \cdots \beta_{n}$ means that $\beta_{i}$ is missing from the expression. That is $\beta_{1} \cdots \beta_{n}$ means $\beta_{1} \cdots \beta_{i-1} \beta_{i+1} \cdots \beta_{n}$, with the particular cases $\beta_{1} \cdots \beta_{n}$ means $\beta_{2} \cdots \beta_{n}$ and $\beta_{1} \cdots \beta_{n}$ means $\beta_{1} \cdots \beta_{n-1}$. This expression is evidently a vector of grade $n-1$. The decretive process reduces the grade. We define further

$$
A_{n-1} \cdot\left(A_{n} \cdot \beta_{1} \cdots \beta_{n}\right) \alpha=(-1)^{n-1} A_{n-1} \cdot \alpha A_{n} \cdot \beta_{1} \cdots \beta_{n} .
$$

We shall see later that we are here following a general law of permutations which is the same as the determinant law for change of sign, but we have to notice that in $A_{0}$ the two vectors may be permuted without change of sign.

If we iterate the process just defined we would have

$$
\begin{aligned}
& A_{n-2} \cdot \alpha_{1} A_{n-1} \cdot \alpha_{2} A_{n} \cdot \beta_{1} \cdots \beta_{n} \\
& \quad=\sum(-1)^{i+j}\left|\begin{array}{cc}
A_{0} \cdot \alpha_{1} \beta_{i} & A_{0} \cdot \alpha_{1} \beta_{j} \\
A_{0} \cdot \alpha_{2} \beta_{i} & A_{0} \cdot \alpha_{2} \beta_{j}
\end{array}\right| A_{n-2} \cdot \beta_{1} \cdots \cdot \beta_{n}, \quad i<j .,
\end{aligned}
$$

This is evident since the multiplier of $A_{n-2} \cdot \beta_{1} \ldots \beta_{n}$ will come from two 
sources; being, if we omit the signs, one way $A_{0} \cdot \alpha_{2} \beta_{j} \cdot A_{0} \cdot \alpha_{1} \beta_{i}$, and the other way $A_{0} \cdot \alpha_{2} \beta_{i} A_{0} \cdot \alpha_{1} \beta_{j}$. But if $i<j$ the sign for the first is $(-)^{i+j+2}$ and for the second $(-)^{i+j+3}$, hence they have opposite signs. It is easy to see by induction that for $r$ iterations we have

$$
\begin{aligned}
A_{n-r} \cdot \alpha_{1} A_{n-r+1} \cdot \alpha_{2} \cdots & A_{n-1} \cdot \alpha_{r} A_{n} \cdot \beta_{1} \cdots \beta_{n} \\
& =\sum(-)^{i_{1}+i_{2}+\cdots+i_{r}+r}\left|A_{0} \cdot \alpha_{k} \beta_{m}\right| A_{n-r} \cdot \beta_{1} \ldots i_{i_{1}} \cdots, i_{r}
\end{aligned}
$$

where $k=1,2, \cdots, r, m=i_{1}, i_{2}, \cdots, i_{r}, i_{1}<i_{2}<\cdots<i_{r}$.

The formula for operation on the right instead of the left is easily written.

As an instance in functions, we may define $A_{n} \cdot \beta_{1} \cdots \beta_{n}$ to be the determinant

$$
\frac{1}{n !} \sum \pm \beta_{1}\left(s_{h_{1}}\right) \beta_{2}\left(s_{h_{2}}\right) \cdots \beta_{n}\left(s_{h_{n}}\right),
$$

a function of $n$ independent variables. If now we multiply by $\alpha_{1}(s)$, we may produce the decretive process by forming the sum, with proper sign, of the integrals we have, when we set in turn $s=s_{1}, s=s_{2}, \cdots, s=s_{n}$.

Since the vectors $\alpha$ enter the expressions above in an alternating manner we are warranted in the final definition, for the decretive process on two vectors of any grades,

$$
\begin{aligned}
A_{n-r} \cdot A_{r} \cdot \alpha_{1} & \cdots \alpha_{r} A_{n} \cdot \beta_{1} \cdots \beta_{n} \\
& =A_{n-r} \cdot \alpha_{1} A_{n-r+1} \cdot \alpha_{2} \cdots A_{n-1} \cdot \alpha_{r} A_{n} \cdot \beta_{1} \cdots \beta_{n}, \quad r \leqq n,
\end{aligned}
$$

and we can show that

$$
A_{n-r} \cdot A_{n} \cdot() A_{r} \cdot()=(-)^{r(n-1)} A_{n-r} \cdot A_{r} \cdot() A_{n} \cdot \text { () when } r \leqq n .
$$

In either case if $r=n$ we have the important formula

$$
\begin{array}{r}
A_{0} \cdot A_{n} \cdot \alpha_{1} \cdots \alpha_{n} A_{n} \cdot \beta_{1} \cdots \beta_{n}=(-)^{\frac{3}{2 n(n-1)}}\left|A_{0} \cdot \alpha_{i} \beta_{j}\right| \\
(i, j=1,2, \cdots, n) .
\end{array}
$$

The expression just found is sometimes called the Grammian when the $\alpha$ 's and $\beta$ 's represent functions. In this case we define

$$
A_{0} \cdot \alpha \beta=\int_{a}^{b} \alpha(s) \beta(s) d s .
$$

For $n$ functions the vanishing of the Grammian is a necessary and sufficient condition of their being linearly dependent. However, this is really because of the presence of the $A_{n}$. of the $n$ functions. It is really what vanishes. It is evident now that we may rewrite the definition of the general decretive process in a theorem

$$
\begin{aligned}
& A_{n-r} \cdot A_{r} \cdot \alpha_{1} \cdots \alpha_{r} A_{n} \cdot \beta_{1} \cdots \beta_{n} \\
& \quad=\sum(-)^{\Sigma i+\frac{1}{2} r(r-1)} A_{0} \cdot A_{r} \cdot \alpha_{1} \cdots \alpha_{r} A_{r} \cdot \beta_{i_{1}} \cdots \beta_{i_{r}} A_{n-r} \beta_{1} \cdots \cdots \cdot \beta_{n} . \cdots, 4
\end{aligned}
$$


It is to be observed that the sign is to be obtained by the determinant rule, since we pass $\beta_{i_{1}}$ over $i_{1}-1$ preceding $\beta$ 's, $\beta_{i_{2}}$ then over $i_{2}-2$ preceding $\beta$ 's, etc. The total inversions are then $i_{1}+i_{2}+\cdots+i_{r}+\frac{1}{2} r(r+1)$. This theorem is a particular case of a more general one to follow, which, however, is to be stated as a definition of both the accretive and the decretive processes for all cases.

The expression $A_{0} \cdot A_{n}$ ( ) $A_{n}$ () is called the norm or square of the intensity of the vector $A_{n}$, as for instance $A_{0} \cdot A_{2} \cdot \alpha \beta A_{2} \cdot \alpha \beta$ is the square of the intensity of $A_{2} \cdot \alpha \beta$. An alternant divided by its intensity, if this is not 0 , gives a unit alternant.

By means of the two processes it is possible to construct the whole of a general vector calculus.*

8. When $A_{0} \cdot A_{n} \cdot \alpha_{1} \cdots \alpha_{n} A_{n} \cdot \beta_{1} \cdots \cdot \beta_{n}=0$, we know from the properties of determinants that if the rank is $h$, by proper addition of rows and columns we may reduce the determinant to one in which either $n-h$ rows or $n-h$ columns contain only 0 , which in the present determinant will give vectors in these rows or columns that are in the complex of the vectors $\alpha$ or the complex of the vectors $\beta$, respectively. It follows that $n-h$ vectors in the complex of $\beta$ 's may be chosen so as to all be orthogonal to every vector $\alpha$, or we may choose $n-h$ vectors in the complex of the $\alpha$ 's which are all orthogonal to all the vectors $\beta$. It follows that the complex of the remaining $h$ linearly independent vectors in the complex of the $\alpha$ 's, and the remaining $h$ linearly independent vectors in the complex of the $\beta$ 's, define two complexes of $h$ dimensions, which have a common incident complex of $h$ dimensions. We must not assume that the complex of order $h$ of the vectors $\alpha$ can be identified with that of order $h$ of the vectors $\beta$, since each vector of the first may be of the form $\sum_{1}^{h} x_{i} \gamma_{i}+\zeta_{j}, j=1, \cdots, h$, and the vectors of the other complex of the form $\sum_{1}^{h} y_{i} \gamma_{i}+\xi_{j}, j=1, \cdots, h$, where the vectors $\zeta$ and $\xi$ are orthogonal to all the vectors $\gamma$, and all vectors $\zeta$ are orthogonal to all vectors $\xi$. The $A_{0}$ process leaves only the bilinear homogeneous forms $\sum x_{i} y_{i}$. It follows that the $h$ vectors $\alpha$ are not necessarily expressible as in the complex of the $h$ vectors $\beta$, and vice versa. We may say then that the complex of the $n$ vectors which determine a vector of grade $n$, and the complex of the $n$ vectors which determine another vector of grade $n$, may have with regard to each other a rank $h$, which is the order of two sub-complexes, one from each complex, which have a common sub-complex of order $h$. In the geometric case it is easy to see the significance of these facts. In the function spaces, however, they are also significant. When the general decretive expression vanishes, each scalar coefficient vanishes provided the vectors $\beta$ are linearly independent. Hence all vectors $\alpha$ and $\beta$ become subject to the remarks just made.

* This was presented to the Society in a paper read March 26, 1921, On Hamiltonian products, second paper. The contents are incorporated in this memoir. 
9. In the alternant $A_{n} \cdot \alpha_{1} \cdots \alpha_{n}$ we may substitute other vectors without changing the value of the alternant. These may be chosen so as to form a mutually orthogonal system if their intensities do not vanish. For it is evident that

$$
\alpha_{2}-\alpha_{1} \frac{A_{0} \cdot \alpha_{1} \alpha_{2}}{A_{0} \cdot \alpha_{1} \alpha_{1}}
$$

is orthogonal to $\alpha_{1}$ and its substitution for $\alpha_{2}$ will not change the value of the alternant. We may now find a vector orthogonal to both $\alpha_{1}, \alpha_{2}$ to substitute for $\alpha_{3}$, etc. In short if we set

$$
\begin{aligned}
& \alpha_{1}^{\prime}=\alpha_{1}, \\
& \alpha_{2}^{\prime}=A_{1} \cdot \alpha_{1} A_{2} \cdot \alpha_{1} \alpha_{2} / A_{0} \cdot \alpha_{1} \alpha_{1}, \\
& \alpha_{3}^{\prime}=A_{1} \cdot A_{2} \cdot \alpha_{1} \alpha_{2} A_{3} \cdot \alpha_{1} \alpha_{2} \alpha_{3} / A_{0} \cdot A_{2} \cdot \alpha_{1} \alpha_{2} A_{2} \cdot \alpha_{1} \alpha_{2},
\end{aligned}
$$

we shall have $A_{n} \cdot \alpha_{1} \cdots \alpha_{n}=A_{n} \cdot \alpha_{1}^{\prime} \cdots \alpha_{n}^{\prime}$.

The process would of course break down in case

$$
A_{0} \cdot \alpha_{1} \alpha_{1}=0, \quad A_{0} \cdot A_{2} \cdot \alpha_{1} \alpha_{2} A_{2} \cdot \alpha_{1} \alpha_{2}=0,
$$

etc. But these are singular vectors and are excluded from the cases that are under consideration. This process of orthogonalizing vectors is of importance in the study of sets of functions, integral equations, etc. If the $A_{0}$ process is defined as in the General Analysis of Moore, so that it is a Hermitian product, the vectors may always be orthogonalized.

10. In case $\rho$ is linearly expressible in terms of $\beta_{1}, \cdots, \beta_{n}$, since we have for any set of $n$ vectors $\alpha_{1}, \cdots, \alpha_{n}$ (writing merely $\alpha$ for $\alpha_{1} \alpha_{2} \cdots \alpha_{n}$ and $\beta$ for $\beta_{1} \beta_{2} \cdots \beta_{n}$ )

$$
\begin{aligned}
A_{1} \cdot A_{n} \cdot \alpha A_{n+1} \cdot \beta \rho=0=( & \left.A_{0} \cdot A_{n} \cdot \alpha A_{n} \beta\right) \rho \\
& -\left(A_{0} \cdot A_{n} \cdot \alpha A_{n} \cdot \beta_{1} \cdots \beta_{n-1} \rho\right) \beta_{n}+\cdots
\end{aligned}
$$

it follows that we have a means of expanding $\rho$ in terms of the vectors $\beta_{1}, \beta_{2}$, $\cdots, \beta_{n}$ if $A_{0} \cdot A_{n} \cdot \alpha A_{n} \cdot \beta \neq 0$; that is, if the set of $\alpha$ 's has rank $n$ with regard to the set of $\beta$ 's. Transposing we have

$\rho=\beta_{n} \frac{A_{0} \cdot \alpha_{n} \cdot A_{n} \cdot \beta_{1} \cdots \beta_{n-1} \rho}{A_{0} \cdot A_{n} \cdot \alpha A_{n} \cdot \beta}$

$$
-\beta_{n-1} \frac{\dot{A_{0}} \cdot A_{n} \cdot \alpha A_{n} \cdot \beta_{1} \cdots \beta_{n-2} \beta_{n} \rho}{A_{0} \cdot A_{n} \cdot \alpha A_{n} \cdot \beta}+\cdots
$$

We now set

$$
\beta^{i}=(-)^{i-1} A_{1} \cdot A_{n-1} \cdot \beta_{1} \cdots \beta_{n} A_{n} \cdot \alpha_{1} \cdots \alpha_{n} / A_{0} \cdot A_{n} \cdot \alpha A_{n} \cdot \beta ;
$$

$\beta^{i}$ is called the complement of $\beta_{i}$ as to the vectors $\alpha$. In case the vectors $\alpha$ 
coincided each to each with the corresponding vectors $\beta$, then the complements would be called the adjuncts of the vectors $\beta$, that is, the adjuncts are complements in the complex of the $\beta$ 's themselves. Evidently

$$
A_{0} \cdot \beta_{i} \beta^{j}=\delta_{i j},
$$

where $\delta_{i j}$ is 1 if $i=j$, and 0 if $i \neq j$. The expression of $\rho$ now becomes

$$
\rho=\sum_{i} \beta_{i} A_{0} \cdot \beta^{i} \rho=\sum_{i} \beta^{i} A_{0} \cdot \beta_{i} \rho .
$$

This is the generalized form for the expansion of $\rho$ commonly used in quaternions.

The process used above for two complexes is called biorthogonalization of the vectors $\beta$ as to the vectors $\alpha$. The complexes need not be the same, but must have their rank with regard to each other equal to the order of the complex. That is to say, the vectors $\alpha$ must be expressible linearly in terms of the common incident complex and other vectors orthogonal to the incident complex. The same is true for the vectors $\beta$. The vectors outside of the incident complex, however, may be quite different. As an example we may show that the sets of vectors

$$
\begin{array}{lcc}
\beta_{1}, \quad A_{1} \cdot \alpha_{1} A_{2} \cdot \beta_{1} \beta_{2}, & A_{1} \cdot A_{2} \cdot \alpha_{1} \alpha_{2} A_{3} \cdot \beta_{1} \beta_{2} \beta_{3}, \\
& A_{1} \cdot A_{3} \cdot \alpha_{1} \alpha_{2} \alpha_{3} A_{4} \cdot \beta_{1} \beta_{2} \beta_{3} \beta_{4}, & \\
\alpha_{1}, \quad A_{1} \cdot \beta_{1} A_{2} \cdot \alpha_{1} \alpha_{2}, & A_{1} \cdot A_{2} \cdot \beta_{1} \beta_{2} A_{3} \cdot \alpha_{1} \alpha_{2} \alpha_{3}, \\
& A_{1} \cdot A_{3} \cdot \beta_{1} \beta_{2} \beta_{3} A_{4} \cdot \alpha_{1} \alpha_{2} \alpha_{3} \alpha_{4}, &
\end{array}
$$

are biorthogonalized sets.

11. We may now give the complete definition that states the result of using either the accretive or the decretive process on two alternants* (counting $A_{1} \cdot \xi$ as an alternant by definition). It could not have been stated before since some of the expressions on the right would have to be defined first. We define for all $i, j$ and any vectors $\alpha, \beta$,

$$
\begin{aligned}
& A_{i+j-2 k} \cdot A_{i} \cdot \alpha_{1} \cdots \alpha_{i} A_{j} \cdot \beta_{1} \cdots \beta_{j} \\
&=\sum(-)^{\Sigma i_{a}+\Sigma j_{b}+\boldsymbol{k}(i-k)} A_{i+j-2 k} \cdot \alpha_{1} \ldots \alpha_{i_{1} \cdots i_{k} j_{1} \cdots j_{k}} \alpha_{i} \beta_{1} \cdots \beta_{j} \\
& \quad \times\left(A_{0} \cdot A_{k} \cdot \alpha_{i_{1}} \cdots \alpha_{i_{k}} A \cdot \beta_{j_{1}} \cdots \beta_{j_{k}}\right)
\end{aligned}
$$

where $\dagger$ we have $k \leqq i, k \leqq j$, that is, $i+j-2 k \geqq|i-j|$. The sign may be determined directly without using the factor containing the sign, by counting the total number of inversions, provided however that in the scalar factor $A_{0} \cdot$ () () the vectors $\alpha$ precede the vectors $\beta$. This definition includes all

* No special name is needed, as the next division shows.

$\dagger$ This $X$ sign merely means that the expression should be written on one line. 
the others given, as particular cases. In case $k \leqq \frac{1}{2} i, i \leqq j$, and $\beta_{i_{a}}=\alpha_{i_{a}}$, $i_{a}=1,2, \cdots, i$, the expression vanishes. In case $k=0$ we have but a single term, the sign being + . If $k=0$ we have the accretive process. If the order of the two alternants is changed it is evident that the result is multiplied by $(-)^{i j+k}$.

The vector just defined, of grade $i+j-2 k$, is the alternant which defines the region or space of order $i+j-2 k$ containing all the regions of the alternants of order $i$ and $j$ determined by omitting common incident regions of order $j$.

\section{The Hamilton product}

12. Hamilton product of two vectors. Let $V_{n}$ and $V_{m}$ be any two vectors of grades $n, m$ respectively, that is, in the complex of a system of alternants all of grade $n$ and the complex of a system of alternants of grade $m$ respectively. Then we define $V_{m+n-2 k} \cdot V_{m} V_{n}=\sum A_{m+n-2 k} \cdot A_{m}^{\prime} A_{n}^{\prime \prime} x_{m} y_{n}$, where $V_{m}=\sum x_{m} A_{m}^{\prime}$, and $V_{n}=\sum y_{n} A_{n}^{\prime \prime}$. This merely states the distributive law. We now define the Hamilton product (also distributive) for two vectors of grades $m, n$, indicating it by the sign ${ }^{*}$ temporarily. We shall see later that no special mark is needed. We remember a scalar is not a vector, though written $A_{0}$. $V_{m}^{*} V_{n}=\sum_{k=0}^{k=|m-n|} V_{m+n-2 k} \cdot V_{m} V_{n}, \quad 0<m, 0<n, \quad V_{m}^{*} x=x V_{m}=x^{*} V_{m}$.

From this definition we have first, since $A_{1} \cdot \alpha_{1}=\alpha_{1}$,

$$
\alpha_{1}^{*} \alpha_{2}=A_{0} \cdot \alpha_{1} \alpha_{2}+A_{2} \cdot \alpha_{1} \alpha_{2} .
$$

Since the two parts of this product are of different grades, there is nothing to interfere with our using the same symbols in front of the Hamilton product as selective symbols to indicate the different parts. That is, we may use

$$
\begin{array}{ll}
A_{0} \cdot \alpha_{1}^{*} \alpha_{2} & \text { and } \\
A_{2} \cdot \alpha_{1} * \alpha_{2} & \text { and } \alpha_{1} \alpha_{2}, \\
A_{2} \cdot \alpha_{1} \alpha_{2}
\end{array}
$$

indifferently. We shall see that this is a procedure which is general.

Again we have

$$
\begin{aligned}
\alpha_{1}{ }^{*} A_{2} \cdot \alpha_{2} \alpha_{3} & =A_{1} \cdot \alpha_{1} A_{2} \cdot \alpha_{2} \alpha_{3}+A_{3} \cdot \alpha_{1} A_{2} \cdot \alpha_{2} \alpha_{3} \\
& =\alpha_{3} A_{0} \cdot \alpha_{1} \alpha_{2}-\alpha_{2} A_{0} \cdot \alpha_{1} \alpha_{3}+A_{3} \cdot \alpha_{1} \alpha_{2} \alpha_{3} .
\end{aligned}
$$

Since $\alpha_{1}^{*} A_{0} \cdot \alpha_{2} \alpha_{3}=\alpha_{1} A_{0} \cdot \alpha_{2} \alpha_{3}$, by adding, we have $\alpha_{1}{ }^{*}\left(\alpha_{2}{ }^{*} \alpha_{3}\right)=\alpha_{1} A_{0} \cdot \alpha_{2} \alpha_{3}-\alpha_{2} A_{0} \cdot \alpha_{1} \alpha_{3}+\alpha_{3} A_{0} \cdot \alpha_{1} \alpha_{2}+A_{3} \cdot \alpha_{1} \alpha_{2} \alpha_{3}$. This product consists then of two vectors, one of grade 1, the other of grade 3 . If we use $A_{1}$ and $A_{3}$ in front of a Hamilton product as selective symbols, then we may define

$$
\begin{aligned}
& A_{1} \cdot \alpha_{1}^{*}\left(\alpha_{2}^{*} \alpha_{3}\right)=\alpha_{1} A_{0} \cdot \alpha_{2} \alpha_{3}-\alpha_{2} A_{0} \cdot \alpha_{1} \alpha_{3}+\alpha_{3} A_{0} \cdot \alpha_{1} \alpha_{2}, \\
& A_{3} \cdot \alpha_{1} *\left(\alpha_{2}{ }^{*} \alpha_{3}\right)=A_{3} \cdot \alpha_{1} \alpha_{2} \alpha_{3} .
\end{aligned}
$$


Evidently

We have

$$
A_{1} \cdot \alpha_{1}^{*}\left(\alpha_{2}^{*} \alpha_{3}\right)=A_{1} \cdot \alpha_{3}^{*}\left(\alpha_{2}^{*} \alpha_{1}\right)
$$

$$
\alpha_{1}^{*}\left(\alpha_{2}^{*} \alpha_{3}\right)=A_{1} \cdot \alpha_{1}^{*}\left(\alpha_{2} * \alpha_{3}\right)+A_{3} \cdot \alpha_{1} \alpha_{2} \alpha_{3}=\left(\alpha_{\mathrm{s}} * \alpha_{2}\right) * \alpha_{3} .
$$

The vector of grade 3 in this product is familiar. The vector of grade 1, however, is a new function of $\alpha_{1}, \alpha_{2}$, and $\alpha_{3}$. We may indicate it as a function of $\alpha_{1}, \alpha_{2}, \alpha_{3}$ by using the same symbol, omitting the multiplication sign*. The alternants we have already written in that manner, the mere juxtaposition of the vectors in $A_{n} \cdot \alpha_{1} \cdots \alpha_{n}$ not implying up to this point anything in the nature of a product. It has meant so far nothing more than a certain defined function $A_{n} \cdot\left(\alpha_{1}, \alpha_{2}, \cdots, \alpha_{n}\right)$ of $n$ vectors, of first grade. Hence we will now define a new system of functions recurrently:

$$
\begin{aligned}
A_{n} \cdot \alpha_{1} \alpha_{2} \cdots \alpha_{n+2 i}=A_{n} \cdot \alpha_{1} & A_{n-1} \cdot \alpha_{2} \cdots \alpha_{n+2 i} \\
& +A_{n} \cdot \alpha_{1} A_{n+1} \cdot \alpha_{2} \cdots \alpha_{n+2 i}, \quad 0<n,
\end{aligned}
$$

where $0 \leqq i$. (The last term vanishes when $i=0$, since $A_{n+1} \cdot \alpha_{2} \cdots \alpha_{n}=0$, for $n+1>n$.)

$$
A_{0} \cdot \alpha_{1} \alpha_{2} \cdots \alpha_{2 m}=A_{0} \cdot \alpha_{1} A_{1} \cdot \alpha_{2} \cdots \alpha_{2 m} .
$$

For instance

$$
A_{1} \cdot \alpha_{1} \alpha_{2} \alpha_{3}=A_{:} \cdot \alpha_{1} A_{0} \cdot \alpha_{2} \alpha_{3}+A_{1} \cdot \alpha_{1} A_{2} \cdot \alpha_{2} \alpha_{3} .
$$

In the case of functional space the number $i$ indicates the number of integrations we perform, there being in all $n+2 i$ parameters which are set equal in $i$ pairs in every possible manner, and then each pair integrated, leaving in every resulting term $n$ parameters as indicated always by the subscript of the $A$. We see that now $A$ has ceased to have only an alternating significance, retaining that meaning when the number of vectors following is the same as the subscript on the $A$. But when the number is greater (it never can be less), then $A$ no longer is an alternating sign. The functions, however, do permit certain permutations depending upon the grade, as will be shown.

13. We shall now prove that the definition just given for $A_{n}$, when the number of vectors exceeds $n$, leads to an expansion in alternants of grade $n$. That is, we shall prove the theorem:

$$
\begin{aligned}
A_{n-2 i} \cdot \alpha_{1} \cdots \alpha_{n}=\sum(-)^{\Sigma j_{a}+i} & A_{0} \cdot \alpha_{j_{1}} \alpha_{j_{2}} \cdots \alpha_{j_{2 i}} \\
& \times A_{n-2 i} \cdot \alpha_{1} \ldots \alpha_{n} \quad(a=1,2, \cdots, 2 i) .
\end{aligned}
$$

As a matter of fact again the signs follow the determinant rule, provided we place the subscripts after $A_{0}$ in natural order, that is, have $j_{1} \leqq j_{2} \leqq \cdots \leqq j_{2 i}$.

For let us suppose this formula holds for all values of $n$ up to and including 
$n=t$, and for all values of $i$, such that $2 i \leqq t$. Then it follows that $A_{1} \cdot \alpha_{1} \alpha_{2} \cdots \alpha_{t}=\alpha_{1} A_{0} \cdot \alpha_{2} \cdots \alpha_{t}-\alpha_{2} A_{0} \cdot \alpha_{1} \cdots \alpha_{t}$

$$
+\cdots+\alpha_{t} A_{0} \cdot \alpha_{1} \cdots \alpha_{t-1}
$$

whenever $t$ is odd, and further when $t$ is odd, so that $t+1$ is even, from the definition in $\S 12$,

$$
\begin{aligned}
A_{0} \cdot \alpha_{1} \alpha_{2} & \cdots \alpha_{t+1} \\
& =A_{0} \cdot \alpha_{1} A_{1} \cdot \alpha_{2} \cdots \alpha_{t+1} \\
& =A_{0} \cdot \alpha_{1} \alpha_{2} A_{0} \cdot \alpha_{3} \cdots \alpha_{t+1}-A_{0} \cdot \alpha_{1} \alpha_{3} A_{0} \cdot \alpha_{2} \alpha_{4} \cdots \alpha_{t+1} \cdots
\end{aligned}
$$

Now by definition whether $t$ is odd or even

$$
\begin{aligned}
A_{t+1-2 j} & =\alpha_{1} \alpha_{2} \cdots \alpha_{t+1} \\
& =A_{t+1-2 j} \cdot \alpha_{1} A_{t+2-2 j} \cdot \alpha_{2} \cdots \alpha_{t+1}+A_{t+1-2 j} \cdot \alpha_{1} A_{t-2 j} \cdot \alpha_{2} \cdots \alpha_{t+1},
\end{aligned}
$$

where $2 j \leqq t+1$. But each term on the right contains a case that comes under the hypothesis since there are only $t$ vectors concerned in the second $A$. Hence we may reduce the right-hand member, and

$$
\begin{aligned}
& A_{t+1-2 j} \cdot \alpha_{1} \alpha_{2} \cdots \alpha_{t+1} \\
& \quad=A_{t+1-2 j} \cdot \alpha_{1} \sum A_{t+2-2 j} \cdot \alpha_{2} \underset{k_{1} \cdots k_{2 j-2}}{\ldots} \alpha_{t+1} A_{0} \cdot \alpha_{k_{1}} \cdots \alpha_{k_{2 j-2}}(-)^{j-1+\Sigma k} \\
& \quad+A_{t+1-2 j} \cdot \alpha_{1} \sum A_{t-2 j} \cdot \alpha_{2} \underset{h_{1} \cdots h_{\mathbf{2}}}{\ldots} \alpha_{t+1} A_{0} \cdot \alpha_{h_{1}} \cdots \alpha_{h_{2 j}}(-)^{j+\Sigma h}
\end{aligned}
$$

where $k_{1}<k_{2}<\cdots<k_{2 j-2}, h_{1}<h_{2}<\cdots<h_{2 j}$.

The first term reduces to

$$
\sum A_{t+1-2 j} \cdot \alpha_{2} \cdots \cdots \alpha_{k_{0} k_{1} \cdots k_{2 j-2}} A_{0} \cdot \alpha_{1} \alpha_{k_{0}} A_{0} \cdot \alpha_{k_{1}} \cdots \alpha_{k_{2 j-2}}(-)^{j-1+\Sigma k+k_{0}+r}
$$

where $r$ is the number of subscripts $k_{1}, k_{2}, \cdots, k_{r}<k_{0}$ in the term, and $k_{0} \neq k_{1}, k_{2}, \cdots, k_{2 j-2}, k_{0}=2,3, \cdots, t+1$ in different terms. This reduces to

$$
\sum A_{t+1-2 j} \cdot \alpha_{2} \alpha_{3} \cdots \cdots \cdot \alpha_{t+1} A_{0} \cdot \alpha_{1} \alpha_{2} \cdots \alpha_{k_{2 j-2}}(-)^{j+1+g+\Sigma k}
$$

in which the subscripts are now rearranged so that $g<k_{1}<k_{2}<\cdots<k_{2 j-2}$. No $k=1$. In these terms $\alpha_{1}$ is missing with $2 j-1$ others, or $2 j$ including $\alpha_{1}$. The second term reduces to

$$
\sum A_{t+1-2 j} \cdot \alpha_{1} \alpha_{2} \cdot \underset{h_{1} \cdots h_{2 j}}{\ldots} \cdot \alpha_{t+1} A_{0} \cdot \alpha_{h_{1}} \cdots \alpha_{h_{2 j}}(-)^{j+\Sigma h}
$$

where no $h$ is 1 , and $h_{1}<h_{2}<\cdots<h_{2 j}$.

In these terms $\alpha_{1}$ is present, and $2 j$ excluding $\alpha_{1}$ are missing. But these two sets of terms together give exactly

$A_{t+1-2 j} \cdot \alpha_{1} \alpha_{2} \cdots \alpha_{t+1}$

$$
=\sum A_{t+1-2 j} \cdot \alpha_{1} \underset{k_{1} \cdots k_{2 j}}{\ldots} \cdot \alpha_{t+1} A_{0} \cdot \alpha_{k_{1}} \cdots \alpha_{k_{2 j}}(-)^{j+\Sigma k}
$$


and hence the theorem holds for the next value of $n=t+1$ and for all values of $j$ that are possible. Since, however, we know the theorem is true for $n=1$, $n=2, n=3$, it holds for ail cases of $n$ an integer. The signs follow the determinant rule provided that under $A_{0}$. the letters are in their natural order, that is, as on the left. We see now from the definition of the Hamilton product of two vectors of any grades that

$$
\begin{array}{r}
\alpha_{1}^{*}\left[A_{n} \cdot \alpha_{2} \cdots \alpha_{n+1}+A_{n-2} \cdot \begin{array}{l}
\alpha_{2} \cdots \alpha_{n+1} \\
\left.\quad+\cdots+A_{1} \text { or } A_{0} \cdot \alpha_{2} \cdots \alpha_{n+1}\right] \\
=A_{n+1} \cdot \alpha_{1} \alpha_{2} \cdots \alpha_{n+1}+A_{n-1} \cdot \alpha_{1} A_{n} \cdot \alpha_{2} \cdots \alpha_{n+1} \\
\quad+\cdots+A_{n-1} \cdot \alpha_{1} A_{n-2} \cdot \alpha_{2} \cdots \alpha_{n+1}+\cdots \\
=A_{n+1} \cdot \alpha_{1} \cdots \alpha_{n+1}+A_{n-1} \cdot \alpha_{1} \cdots \alpha_{n+1} \\
+\cdots+A_{0} \text { or } A_{1} \cdot \alpha_{1} \cdots \alpha_{n+1} .
\end{array}\right.
\end{array}
$$

Now we know already that

$$
\alpha_{n-1} * \alpha_{n}=A_{2} \cdot \alpha_{n-1} \alpha_{n}+A_{0} \cdot \alpha_{n-1} \alpha_{n}
$$

and

$$
\alpha_{n-2} *\left(\alpha_{n-1} * \alpha_{n}\right)=A_{3} \cdot \alpha_{n-2} \alpha_{n-1} \alpha_{n}+A_{1} \cdot \alpha_{n-2} \alpha_{n-1} \alpha_{n} .
$$

It is evident from the theorem just proved that we must have the general Hamilton product (in which the multiplier is set at the left) given by the theorem

$$
\alpha_{1}^{*}\left[\alpha_{2}^{*}\left(\cdots * \alpha_{n}\right)\right]=A_{n} \cdot \alpha_{1} \cdots \alpha_{n}+A_{n-2} \cdot \alpha_{1} \cdots \alpha_{n}+\cdots .
$$

If the $A$ 's are inserted in front of this product they are understood as selective symbols that pick out the term on the right of corresponding grade. We have now to show that this product is associative, that is, we may group the vectors in the order in which they come, in any manner, and find that the Hamilton product of the groups is the Hamilton product of the whole set. For instance $\left[\alpha_{1}^{*}\left(\alpha_{2}^{*} \alpha_{3}\right)\right]^{*}\left(\alpha_{4}^{*} \alpha_{5}\right)=\alpha_{1} *\left[\left(\alpha_{2} * \alpha_{3}\right) *\left(\alpha_{4} * \alpha_{5}\right)\right]=\alpha_{1} *\left[\alpha_{2} *\left(\alpha_{3}^{*} \overline{\alpha_{4} * \alpha_{5}}\right)\right]$.

14. Associativity of the Hamilton product. We shall prove this by showing that whether we multiply successively $\alpha_{n}$ by $\alpha_{n-1}$, then $\alpha_{n-2}, \cdots$, by $\alpha_{1}$, or whether we form the product of $\alpha_{n}$ by $\alpha_{n-1}, \cdots$, by $\alpha_{i}$, and then the product of this by the product of $\alpha_{i}$ multiplied successively by $\alpha_{i-1}, \alpha_{i-2}, \cdots$, by $\alpha_{1}$, we have the same result. That is, we are to prove that

$$
\begin{aligned}
& {\left[A_{i} \cdot \alpha_{1} \cdots \alpha_{i}+A_{i-2} \cdot \alpha_{1} \cdots \alpha_{i}+\cdots\right]} \\
& \quad *\left[A_{n-i} \cdot \alpha_{i+1} \cdots \alpha_{n}+A_{n-i-2} \cdot \alpha_{i+1} \cdots \alpha_{n}\right] \\
& \quad=A_{n} \cdot \alpha_{1} \cdots \alpha_{n}+A_{n-2} \cdot \alpha_{1} \cdots \alpha_{n}+\cdots .
\end{aligned}
$$

In the first place let $n=i+j$. Then

$$
A_{i-2 q} \cdot \alpha_{1} \cdots \alpha_{i}=\sum(-)^{\Sigma i_{x}+q} A_{i-2 q}\left(i_{1}, \cdots, i_{2 q}\right) A_{0} \cdot \alpha_{i_{1}} \cdots \alpha_{i_{2 q}},
$$


where the subscripts written inside the parenthesis will indicate the missing vectors from $\alpha_{1}, \cdots, \alpha_{i}$. Also we have

$$
\begin{aligned}
& A_{j-2 m} \alpha_{i+1} \cdots \alpha_{i+j}=\sum(-)^{\Sigma j_{x}+m} A_{j-2 m}\left(j_{1}, \cdots, j_{2 m}\right) A_{0} \cdot \alpha_{j_{1}} \cdots \alpha_{j_{2 m}}, \\
& A_{i+j-2(m+p+q)} \cdot A_{i-2 q} \cdot \alpha_{1} \cdots \alpha_{i} A_{j-2 m} \cdot \alpha_{i+1} \cdots \alpha_{i+j} \\
& =\sum(-)^{\Sigma i_{x}+\Sigma j_{z}+q+m} A_{i+j-2(m+p+q)} A_{i-2 q}\left(i_{1}, \cdots, i_{2 q}\right) \\
& \quad \times A_{j-2 m}\left(j_{1}, \cdots, j_{2 m}\right) A_{0} \cdot \alpha_{i_{1}} \cdots \alpha_{i_{2 q}} A_{0} \cdot \alpha_{j_{1}} \cdots \alpha_{j_{2 m}} \\
& =\sum(-)^{\Sigma i_{x}+\Sigma j_{2}+q+m+p} A_{i+j-2(m+p+q)} \alpha_{1} \alpha_{2} \cdots \cdots i_{i_{1} \cdots i_{2 q+p} j_{1} \cdots j_{2 m+p}} \\
& \quad \times A_{i+j} \cdot \alpha_{i_{1}} \cdots \alpha_{i_{2 q}} A_{0} \cdot \alpha_{j_{1}} \cdots \alpha_{j_{2 m}} A_{0} \\
& \quad A_{p} \alpha_{i_{2 q+1}} \cdots \alpha_{i_{2 q+p}} A_{p} \cdot \alpha_{j_{2 m+1}} \cdots \alpha_{j_{2 m+p}} .
\end{aligned}
$$

Let $r=m+p+q$; then we may condense the expression just written into $\sum(-)^{r} A_{i+j-2 r}\left(i_{1} \cdots i_{2 q+p} j_{1} \cdots j_{2 m+p}\right) A_{0} \cdot A_{p} \cdot \alpha_{i_{1}}$

$$
\cdots \alpha_{i_{2 q+p}} A_{p} \cdot \alpha_{j_{1}} \cdots \alpha_{j_{2 m+p}} \text {. }
$$

If now we set $q+m=h, p=r-h$, and holding $h$ fixed let $q$ and $m$ take all possible values, we have from the above

$$
\begin{aligned}
\sum_{h=0}^{h=r} \sum_{l=0, m=h}^{l=h_{1} m=0} A_{i+j-2 r} \cdot A_{i-2 l} \cdot \alpha_{1} \cdots \alpha_{i} A_{j-2 m} \alpha_{i+1} \cdots \alpha_{i+j} \\
=\sum_{h}(-)^{r} A_{i+j-2 r} \alpha_{1} \cdot \ldots \cdot \alpha_{i} \alpha_{i+1} \cdot \ldots \cdot \alpha_{j} A_{0} \cdot A_{r-h} \cdot \alpha_{i_{1}} \\
\cdots i_{1} \cdots j_{t} \\
\cdots \alpha_{i_{s}} A_{r-h} \cdot \alpha_{j_{1}} \cdots \alpha_{j_{t}},
\end{aligned}
$$

where we must have $s+t=2 r$ and $r-h \leqq s \leqq r+h$. But finally summing as to $h$, we have exactly the sum

$$
\sum_{r} A_{i+j-2 r} \alpha_{1} \cdots \alpha_{i} \alpha_{i+1} \cdots \alpha_{i+j},
$$

which is the expansion of the Hamiltonian product of $\alpha_{1}, \alpha_{2}, \cdots, \alpha_{i+j}$ in the order written. On the left the final sums give the Hamiltonian products of $\alpha_{1}, \alpha_{2}, \cdots, \alpha_{i}$ and $\alpha_{i+1}, \cdots, \alpha_{i+j}$.

We see now that the special use of a multiplication sign is unnecessary and that in all the functions we have built up, with the use of the $A$ and subscripts, the array of vectors may be considered to be a Hamilton product, and the $A$ a selective sign for the vector of the indicated grade. The remarkable simplicity of this result enables us to dispense with any very large collection of formulas for transforming expressions in this general vector calculus. We may break up by associativity any expression under an $A$ and arrive at various reductions in this way. As instances

$$
\begin{aligned}
& \begin{array}{l}
A_{0} \cdot \alpha_{1} \alpha_{2} \alpha_{3} \alpha_{4} \\
\alpha_{5} \alpha_{6}=A_{0} \cdot\left(\alpha_{1} \alpha_{2} \alpha_{3} \alpha_{4}\right)\left(\alpha_{5} \alpha_{6}\right) \\
=A_{0} \cdot\left[\left(A_{4}+A_{2}+A_{0}\right) \cdot \alpha_{1} \alpha_{2} \alpha_{3} \alpha_{4}\right]\left[A_{2}+A_{0}\right] \cdot \alpha_{5} \alpha_{6} \\
=A_{0} \cdot A_{2} \cdot \alpha_{1} \alpha_{2} \alpha_{3} \alpha_{4} A_{2} \cdot \alpha_{5} \alpha_{6}+A_{0} \cdot \alpha_{1} \alpha_{2} \alpha_{3} \alpha_{4} A_{0} \cdot \alpha_{5} \alpha_{6} ;
\end{array} \\
& \begin{array}{l}
A_{2} \cdot\left(A_{2} \cdot \alpha_{1} \alpha_{2} A_{4} \cdot \beta_{1} \beta_{2} \beta_{3} \beta_{4}\right)
\end{array}
\end{aligned}
$$$$
=A_{2}\left(\alpha_{1} \alpha_{2}-A_{0} \cdot \alpha_{1} \alpha_{2}\right) A_{4} \cdot \beta_{1} \beta_{2} \beta_{3} \beta_{4}=A_{2} \cdot\left(\alpha_{1} \alpha_{2} A_{4} \cdot \beta_{1} \beta_{2} \beta_{3} \beta_{4}\right) \text {; }
$$

$A_{0} \cdot \alpha_{1} \alpha_{2} \alpha_{3} \alpha_{4}=A_{0} \cdot \alpha_{1} \alpha_{2} A_{0} \cdot \alpha_{3} \alpha_{4}$

Trans. Am. Math. Soc. 15.

$$
-A_{0} \cdot \alpha_{1} \alpha_{3} A_{0} \cdot \alpha_{2} \alpha_{4}+A_{0} \cdot \alpha_{1} \alpha_{4} A_{0} \cdot \alpha_{2} \alpha_{3}
$$


Expansions of this sort are unlimited in number.* Other useful formulas are easily derived by combinations, as

$$
\begin{aligned}
& \begin{array}{l}
A_{n} \cdot \alpha A_{n-1} \beta A_{n} \cdot \gamma_{1} \cdots \gamma_{n}+A_{n} \cdot \beta A_{n+1} \cdot \alpha A_{n} \cdot \gamma_{1} \cdots \gamma_{n} \\
=A_{n} \cdot \alpha \beta A_{n} \cdot \gamma_{1} \cdots \gamma_{n}
\end{array} \\
& \begin{array}{r}
A_{1} \cdot A_{n} \cdot \alpha_{1} \cdots \alpha_{n} A_{n+1} \rho \beta_{1} \cdots \beta_{n}=(-)^{n} \rho A_{0} \cdot A_{n} \cdot \alpha_{1} \\
\cdots \alpha_{n} A_{n} \cdot \beta_{1} \cdots \beta_{n}-\sum \beta_{j} A_{0} \cdot A_{n} \cdot \alpha_{1} \cdots \alpha_{n} A_{n} \cdot \rho \beta_{1} \cdots \beta_{n}
\end{array} \\
& \begin{array}{r}
A_{1} \cdot \alpha_{1} A_{2} \cdot \alpha_{2} \alpha_{3}+A_{1} \cdot \alpha_{2} A_{2} \cdot \alpha_{3} \alpha_{1}+A_{1} \cdot \alpha_{3} A_{2} \cdot \alpha_{1} \alpha_{2}=0 \\
A_{2} \cdot \alpha_{1} A_{3} \cdot \alpha_{2} \alpha_{3} \alpha_{4}-A_{2} \cdot \alpha_{2} A_{3} \cdot \alpha_{1} \alpha_{3} \alpha_{4} \\
+A_{2} \cdot \alpha_{3} A_{3} \cdot \alpha_{1} \alpha_{2} \alpha_{4}-A_{2} \cdot \alpha_{4} A_{3} \cdot \alpha_{1} \alpha_{2} \alpha_{3}=0
\end{array}
\end{aligned}
$$

15. There are other ways by which we might have developed the subject. We might have taken a method similar to that of Clifford and defined the symbols $\alpha, \beta$, etc., which we have called vectors, as the hypernumbers defined by the ratios of geometric vectors; but this method (and others) has great disadvantages in the end. We might of course have defined a general or indeterminate product and derived from this expressions which would have been our $A$ expressions. Some of these will be exhibited later. We should have difficulties also this way. From a long consideration of the problem the method of development used was settled on as the most desirable from a good many points of view.

16. We proceed to develop a few theorems useful in applications. If we consider two complexes of order $n$ each, defined by $\alpha_{1}, \cdots, \alpha_{n}$ and $\beta_{1}, \cdots, \beta_{n}$, and a vector $\rho$, the part of $\rho$ in the incident complex of the two (say $\rho^{\prime}$ ) is given by

$$
\rho^{\prime} A_{0} \cdot A_{n} \cdot \alpha A_{n} \cdot \beta=A_{1} \cdot\left(A_{n-1} \cdot \rho A_{n} \alpha\right) A_{n} \cdot \beta .
$$

For it is easy to see that

$$
A_{n-1} \cdot \rho A_{n} \cdot \alpha_{1} \cdots \alpha_{n}=\sum A_{0} \cdot \alpha_{i} \rho A_{n-1} \cdot \alpha_{1} \cdots \alpha_{n}(-)^{i-1},
$$

and therefore the right side gives $\sum_{i} A_{0} \cdot \alpha_{i} \rho \cdot \alpha^{i} \cdot A_{0} \cdot A_{n} \cdot \alpha A_{n} \cdot \beta$, when we set for the complement of $\alpha_{i}$

$$
\alpha^{i}=(-)^{i-1} \cdot A_{1} \cdot A_{n-1} \cdot \alpha_{1} \cdots \alpha_{n} A_{n} \cdot \beta_{1} \cdots \beta_{n} / A_{0} \cdot A_{n} \cdot \alpha A_{n} \cdot \beta
$$

But the form we now have is that part of $\rho$ which lies in the complex of $\alpha^{1}, \alpha^{2}$, $\cdots, \alpha^{n}$, that is, in the incident complex. It follows that

$$
\rho-\frac{A_{1} \cdot\left(A_{n-1} \cdot \rho A_{n} \cdot \alpha\right) A_{n} \cdot \beta}{A_{0} \cdot A_{n} \cdot \alpha A_{n} \cdot \beta}
$$

* Conversely every expression $A_{m} \cdot \alpha_{1} \cdots \alpha_{n}$, may be expressed as a sum of products alone without any $A$ 's. See page 220 . 
is the part of $\rho$ (its projection) exterior to the incident space. It may be also written in the form

$$
\frac{A_{1} \cdot\left[\rho A_{0} \cdot A_{n} \cdot \alpha A_{n} \cdot \beta-\left(A_{n-1} \cdot \rho A_{n} \cdot \alpha\right) A_{n} \cdot \beta\right]}{A_{0} \cdot A_{n} \cdot \alpha A_{n} \cdot \beta} .
$$

17. Another useful form is the following. Since $A_{1} \cdot A_{n-1} \cdot \beta_{1} \cdots \beta_{n-1} A_{n} \cdot \alpha$ is linear in $\alpha_{1}, \cdots, \alpha_{n}$, we see that if we consider a set of vectors defining a complex of order $r, \alpha_{1}, \cdots, \alpha_{n}, \alpha_{n+1}, \cdots, \alpha_{r}, n \leqq r$, then

$$
A_{r+1} \cdot\left[A_{1} \cdot A_{n-1} \cdot \beta_{1} \cdots \beta_{n-1} A_{n} \cdot \alpha_{1} \cdots \alpha_{n}\right] A_{r} \cdot \alpha_{1} \cdots \alpha_{r}=0
$$

identically. This is true whatever the vectors $\beta$. Hence for any vectors $\gamma_{1}, \cdots, \gamma_{r+1}$

$A_{0} \cdot\left[A_{r+1} \cdot \gamma_{1} \cdots \gamma_{r+1} A_{r+1} \cdot A_{1}\left(A_{n-1} \cdot \beta_{1}\right.\right.$ $\left.\left.\cdots \beta_{n-1} A_{n} \cdot \alpha_{1} \cdots \alpha_{n}\right) A_{r} \cdot \alpha_{1} \cdots \alpha_{r}\right]=0$

identically. We may now change the order and have still identically

$A_{0} \cdot\left[A_{r+1} \cdot\left(A_{r} \cdot \alpha_{1} \cdots \alpha_{r} A_{1} \cdot A_{n-1} \cdot \beta_{1}\right.\right.$

$$
\left.\left.\cdots \beta_{n-1} A_{n} \cdot \alpha_{1} \cdots \alpha_{n}\right) A_{r+1} \cdot \gamma_{1} \cdots \gamma_{r+1}\right]=0 .
$$

We may however drop the first $A_{r+1}$ since $A_{0}$ in front prevents any additional terms from entering, and then for the same reason we may insert $A_{r}$ as shown, arriving at a formula from which many useful formulas may be deduced: *

$A_{0} \cdot A_{r} \cdot \alpha_{1} \cdots \alpha_{r} A_{r} \cdot\left[A_{1} \cdot\left(A_{n-1} \cdot \beta_{1}\right.\right.$

$$
\left.\left.\cdots \beta_{n-1} A_{n} \cdot \alpha_{1} \cdots \alpha_{n}\right) A_{r+1} \cdot \gamma_{1} \cdots \gamma_{r+1}\right]=0 \text {. }
$$

18. A similar formula may be deduced from

$$
A_{x} \cdot A_{y} \cdot \beta_{1} \cdots \beta_{y} A_{x+y} \cdot \alpha_{1} \cdots \alpha_{x+y},
$$

which is linear in $A_{x} \cdot \alpha_{i_{1}} \cdots \alpha_{i_{x}}$. If then we consider the set $\alpha_{1}, \cdots, \alpha_{x+y+z}$ where $z$ may be zero, we have the identity

$A_{2 x+y+z} \cdot\left(A_{x} \cdot A_{y} \cdot \beta_{1} \cdots \beta_{y} A_{x+y} \cdot \alpha_{1} \cdots \alpha_{x+y}\right) A_{x+y+z} \cdot \alpha_{1} \cdots \alpha_{x+y+z}=0$.

Hence whatever the vectors $\gamma_{1}, \cdots, \gamma_{2 x+y+z}$, we have identically

$A_{0} \cdot\left[A_{2 x+y+z} \cdot \gamma_{1} \cdots \gamma_{2 x+y+z} A_{2 x+y+z}\right.$

- $\left.\left(A_{x} \cdot A_{y} \cdot \beta_{1} \cdots \beta_{y} A_{x+y} \cdot \alpha_{1} \cdots \alpha_{x+y}\right) A_{x+y+z} \cdot \alpha_{1} \cdots \alpha_{x+y+z}\right]=0$,

whence for all $\beta$ 's and $\gamma$ 's we have

$A_{0} \cdot\left[A_{x+y+z} \cdot \alpha_{1} \cdots \alpha_{x+y+z} A_{x}\right.$

$$
\left.\cdot\left(A_{y} \cdot \beta_{1} \cdots \beta_{y} A_{x+y} \cdot \alpha_{1} \cdots \alpha_{x+y}\right)\right] A_{2 x+y+z} \cdot \gamma_{1} \cdots \gamma_{2 x+y+z}=0 \text {. }
$$

Particular cases occur for $z=0$.

*Volterra, Rice Institute Pamphlets, vol. 4 (1917), No. 1, p. 58 (3). 
19. In the expression

$$
\begin{aligned}
& A_{2 r+s-2 x} \cdot A_{r} \cdot \alpha_{1} \cdots \alpha_{r} A_{r+s} \cdot \alpha_{1} \cdots \alpha_{r} \beta_{1} \cdots \beta_{s} \\
& =\sum \pm A_{0} \cdot A_{x} \cdot \alpha_{i_{1}} \cdots \alpha_{i_{x}} A_{x} \cdot \alpha_{j_{1}} \cdots \alpha_{j_{y}} \beta_{k_{1}}
\end{aligned}
$$

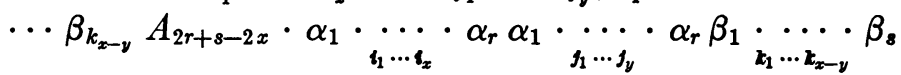

the vectors $\alpha$ that enter the right-hand side are all different, and there are not more than $r$, hence we have $2 r-x-y \leqq r$ or $r \leqq x+y$. Further if the $j$ set and the $i$ set do not together make up the whole $r$ set, then there are inevitably duplicates in the last factor which would cause it to vanish. Hence the $i$ set and the $j$ set must cover all the $r$ vectors $\alpha_{1}, \cdots, \alpha_{r}$, though they may overlap, in which case in the last factor the overlap would be missing. In each $A$ we may reduce with no alteration in value by substituting an orthogonal set for the vectors appearing there (indicated by accents). The factor $A_{0}$ would then vanish if it contained any vectors $\beta$. Hence the vectors of the $i$ set and those of the $j$ set must define the same complex. They must therefore each be the whole set $\alpha_{1}, \cdots, \alpha_{r}$. Hence for any value except $x=y=r$ the expression vanishes, and when $x=y=r$ it reduces to

$$
A_{s} \cdot \beta_{1}^{\prime} \cdots \beta_{s}^{\prime} \text {. }
$$

20. If we consider the bordered determinant

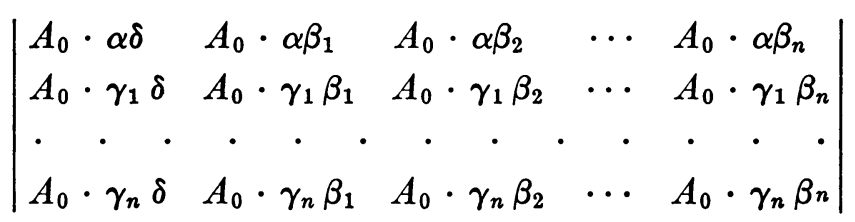

we see that it may be condensed into

$$
(-)^{\operatorname{m}(n+1)} A_{0} \cdot A_{n+1} \cdot \delta \beta_{1} \beta_{2} \cdots \beta_{n} A_{n+1} \cdot \alpha \gamma_{1} \gamma_{2} \cdots \gamma_{n} .
$$

If we expand by Cauchy's theorem the determinant is equal to

$$
\Delta \cdot A_{0} \cdot \alpha \delta-\sum A_{0} \cdot \alpha \beta_{i} A_{0} \cdot \delta \gamma_{j} \cdot \Delta_{i j},
$$

where $\Delta$ is the determinant of $\beta$ and $\gamma$ alone, that is, the one bordered, and $\Delta_{i j}$ is the minor of $\Delta$ produced by omitting the column containing $\beta_{i}$ and row containing $\gamma_{j}$. Now interchange $\alpha$ and $\delta$ and subtract. The first terms cancel and the others become

$$
\begin{aligned}
\sum A_{0} \cdot A_{2} \cdot \alpha \delta A_{2} \cdot \beta_{i} \gamma_{j} \cdot \Delta_{i j}=\sum A_{0} \cdot A_{2} \cdot \alpha \delta A_{2} \\
\cdot \beta_{i} \gamma_{j} A_{0} \cdot A_{n-1} \cdot \beta_{1} \ldots \beta_{n} A_{n-1} \cdot \gamma_{1} \cdots \gamma_{j}(-)^{\frac{3}{3(n-1)(n-2)+i+j}} .
\end{aligned}
$$

This is easily reduced to

$$
(-)^{\mathbf{3}(n-1) n+1} A_{0} \cdot A_{2} \cdot \alpha \delta A_{2} \cdot A_{n} \cdot \beta_{1} \cdots \beta_{n} A_{n} \cdot \gamma_{1} \cdots \gamma_{n} .
$$


Hence we have the useful theorem

$$
\begin{aligned}
A_{0} \cdot A_{n+1} \cdot \alpha \beta_{1} \cdots \beta_{n} A_{n+1} \cdot \delta \gamma_{1} \cdots \gamma_{n}-A_{0} \cdot A_{n+1} \cdot \delta \beta_{1} \\
\cdots \beta_{n} A_{n+1} \cdot \alpha \gamma_{1} \cdots \gamma_{n}=(-)^{n} A_{0} \cdot A_{2} \cdot \alpha \delta A_{2} \cdot A_{n} \cdot \beta A_{n} \cdot \gamma
\end{aligned}
$$

21. We notice that

$$
\begin{aligned}
A_{n} \cdot \beta_{1} \cdots \beta_{n}{ }^{*} \alpha & =A_{n+1} \cdot\left(A_{n} \cdot \beta_{1} \cdots \beta_{n} * \alpha\right)+A_{n-1} \cdot\left(A_{n} \cdot \beta_{1} \cdots \beta_{n} * \alpha\right) \\
& =(-)^{n}\left[A_{n+1} \cdot \alpha \beta_{1} \cdots \beta_{n}-A_{n-1} \cdot \alpha A_{n} \cdot \beta_{1} \cdots \beta_{n}\right] .
\end{aligned}
$$

Hence we have since $A_{n+2} \cdot \alpha A_{n+1} \alpha()=0$, and $A_{n-2} \cdot \alpha A_{n-1} \cdot \alpha()=0$,

$$
\begin{aligned}
\alpha^{*} & A_{n} \cdot \beta_{1} \cdots \beta_{n} * \alpha \\
= & (-)^{n}\left[A_{n} \cdot \alpha A_{n+1} \cdot \alpha \beta_{1} \cdots \beta_{n}-A_{n} \cdot \alpha A_{n-1} \cdot \alpha A_{n} \cdot \beta_{1} \cdots \beta_{n}\right] \\
= & (-)^{n}\left[A_{0} \cdot \alpha \alpha A_{n} \cdot \beta_{1} \cdots \beta_{n}-2 A_{0} \cdot \alpha \beta_{1} \cdot A_{n} \cdot \alpha \beta_{2}\right. \\
& \left.\cdots \beta_{n}+2 A_{0} \cdot \alpha \beta_{2} A_{n} \cdot \alpha \beta_{1} \beta_{3} \cdots \beta_{n}\right] \\
& =A_{n} \cdot \alpha \beta_{1} \cdots \beta_{n} \alpha .
\end{aligned}
$$

It is apparent then that if we multiply an alternant of grade $n$ by the same linear vector on the right and left, we have a vector of grade $n$, which is the part of grade $n$ of the product of the original product by this vector on the right and the left.

Since we have $A_{0} \cdot \alpha \beta_{1} \cdots \beta_{n} \alpha=A_{0} \cdot \alpha \alpha A_{0} \cdot \beta_{1} \cdots \beta_{n}$ and since

$$
\alpha \alpha=A_{0} \cdot \alpha \alpha \text {, and } \alpha \cdot A_{0} \cdot \beta_{1} \cdots \beta_{n} \cdot \alpha=\alpha \alpha A_{0} \cdot \beta_{1} \cdots \beta_{n},
$$

it is evident that for any expression

$$
A_{m} \cdot \beta_{1} \cdots \beta_{n}
$$

we have

$$
\alpha^{*} A_{m} \cdot \beta_{1} \cdots \beta_{n}^{*} \alpha=A_{m} \cdot \alpha \beta_{1} \cdots \beta_{n} \alpha .
$$

A vector of grade 1 which comes at both extremes of any vector of any grade of a product may be set outside the vector symbol of that grade. This enables us to make reductions. For instance

$$
\begin{aligned}
\alpha \beta \gamma \alpha & =\alpha A_{2} \cdot \beta \gamma \cdot \alpha+\alpha \alpha A_{0} \cdot \beta \gamma ; \\
\alpha \beta \gamma \alpha \delta & =\alpha \alpha A_{0} \cdot \beta \gamma \cdot \delta+\alpha A_{2} \cdot \beta \gamma \cdot \alpha \delta .
\end{aligned}
$$

This theorem is useful in studying invariancy under rotation about linear vectors. The papers of Joly and M'Aulay should be consulted.

It is evident that continued application of the theorem is possible. For instance

$$
\left(\alpha_{1} \cdots \alpha_{n}\right) V_{m} \cdot \beta_{1} \cdots \beta_{r}\left(\alpha_{n} \cdots \alpha_{1}\right)=V_{m} \cdot \alpha_{1} \cdots \alpha_{n} \beta_{1} \cdots \beta_{r} \alpha_{n} \cdots \alpha_{1} .
$$

22. Since the formulas we are to consider in this section are homogeneous in all vectors on both sides, we will consider that the vectors are unit vectors, that is to say for each vector we have

$$
\xi \xi=A_{0} \cdot \xi \xi=1
$$


We may introduce multipliers easily to produce vectors not unit vectors. We will occasionally indicate the opposite of a vector by a stroke over the symbol, thus

$$
-\alpha=\bar{\alpha}
$$

We have then $\alpha \bar{\alpha}=-1$. We shall use the stroke extended to cover more than one vector to indicate that the order is to be reversed and all the signs, thus

$$
\overline{\alpha_{1} \cdots \alpha_{n}}=\bar{\alpha}_{n} \cdots \bar{\alpha}_{1} .
$$

This is called, in quaternions, taking the conjugate.

This operation will not affect the form $A_{0} \cdot \alpha_{1} \cdots \alpha_{n}$, for this vanishes when $n$ is odd, and if $n=2 m$ we have by definition of $A_{0} \cdot \alpha_{1} \cdots \alpha_{2 m}$

$$
A_{0} \cdot \alpha_{1} \cdots \alpha_{2 m}=\sum(-)^{p} A_{0} \cdot \alpha_{i_{1}} \alpha_{j_{1}} A_{0} \cdot \alpha_{i_{2}} \alpha_{j_{2}} \cdots A_{0} \cdot \alpha_{i_{m}} \alpha_{j_{m}} \text {, }
$$

where the subscripts are so arranged that $i_{x}<j_{x}(x=1,2, \cdots, m)$ and $p$ is the number of inversions in the total collection of subscripts so arranged. It simplifies the count a little to arrange either the subscripts $i$ or $j$ in their natural order. It is evident now that as each factor may have its vectors interchanged, and as a change of both signs does not affect the value, we will have in any case

$$
A_{0} \cdot \overline{\alpha_{1} \cdots \alpha_{n}}=A_{0} \cdot \alpha_{1} \cdots \alpha_{n} .
$$

Hence if we remember the expansion of the form $A_{m} \cdot \alpha_{1} \cdots \alpha_{n}$, we see that as every term is an alternant of grade $m$, and as

$$
A_{m} \cdot \overline{\beta_{1} \cdots \beta_{m}}=(-)^{m} A_{m} \cdot \beta_{m} \cdots \beta_{1}=(-)^{\text {tm(m+1) }} A_{m} \cdot \beta_{1} \cdots \beta_{m},
$$

we shall have

$$
A_{m} \cdot \overline{\alpha_{1} \cdots \alpha_{n}}=(-)^{\frac{1}{3 m(m+1)}} A_{m} \cdot \alpha_{1} \cdots \alpha_{n} .
$$

Hence we have the four forms for any product $\pi=\alpha_{1} \cdots \alpha_{n}$, according to form of $n$,

$$
\begin{aligned}
A_{4 m} \cdot \bar{\pi} & =A_{4 m} \cdot \pi ; & -A_{4 m+1} \cdot \bar{\pi} & =A_{4 m+1} \cdot \pi ; \\
-A_{4 m+2} \cdot \bar{\pi} & =A_{4 m+2} \cdot \pi ; & A_{4 m+3} \cdot \bar{\pi} & =A_{4 m+3} \cdot \pi .
\end{aligned}
$$

It follows that

$$
\bar{\pi}=\left(A_{0} \cdot-A_{1} \cdot-A_{2} \cdot+A_{3} \cdot+A_{4} \cdot-A_{5} \cdot \cdots\right) \cdot \pi .
$$

Hence

$$
\begin{array}{ll}
\frac{1}{2}(\pi+\bar{\pi})=\left(A_{0} \cdot+A_{4} \cdot+\cdots\right) \pi & \text { if } \pi \text { is of even grade, } \\
\frac{1}{2}(\pi-\bar{\pi})=\left(A_{2} \cdot+A_{6} \cdot+\cdots\right) \pi & \text { if } \pi \text { is of even grade, } \\
\frac{1}{2}(\pi+\bar{\pi})=\left(A_{1} \cdot+A_{5} \cdot+\cdots\right) \pi & \text { if } \pi \text { is of odd grade, } \\
\frac{1}{2}(\pi-\bar{\pi})=\left(A_{3}+A_{7}+\cdots\right) \pi & \text { if } \pi \text { is of odd grade. }
\end{array}
$$


We may also state the theorem thus: ${ }^{*}$ in $A_{0}, A_{4}, \cdots$ we may reverse the order of the product, in $A_{1}, A_{5}, \cdots$ we may reverse the order, in $A_{2}, A_{6}, \cdots$ and in $A_{3}, A_{7}, \ldots$ we must change the sign if we reverse the order. We may also state it thus: the substitution $(1, n)(2, n-1)(3, n-2) \cdots$ leaves invariant $A_{0}, A_{4}, \cdots, A_{1}, A_{5}, \cdots$ and changes the sign of $A_{2}, A_{6}, \cdots, A_{3}, A_{7}, \cdots$.

Again we notice that if $\mu$ is a vector of grade $m$, then

$$
\mu \alpha=A_{m+1} \cdot \mu \alpha+A_{m-1} \cdot \mu \alpha, \quad \alpha \mu=A_{m+1} \cdot \alpha \mu+A_{m-1} \cdot \alpha \mu,
$$

since all other grades vanish, and by the formula in $\S 11$ we have when we interchange the vectors

$$
\begin{aligned}
& \alpha \mu=(-)^{m}\left[A_{m+1} \cdot \mu \alpha-A_{m-1} \cdot \mu \alpha\right], \\
& \mu \alpha=(-)^{m}\left[A_{m+1} \cdot \alpha \mu-A_{m-1} \cdot \alpha \mu\right] .
\end{aligned}
$$

This gives us at once

$$
\begin{aligned}
& \mu \alpha+(-)^{m} \alpha \mu=2 A_{m+1} \cdot \mu \alpha=(-)^{m} 2 A_{m+1} \cdot \alpha \mu, \\
& \mu \alpha-(-)^{m} \alpha \mu=2 A_{m-1} \cdot \mu \alpha=(-)^{m-1} 2 A_{m-1} \cdot \alpha \mu .
\end{aligned}
$$

We have from this, reverting to $\pi=\alpha_{1} \cdots \alpha_{n}$, and writing $\pi_{s}=A_{s} \cdot \pi \cdot$ for brevity,

$$
\pi \alpha=\sum \pi_{s} \alpha=\sum(-)^{s}\left[A_{s+1} \cdot \alpha \pi_{s}-A_{s-1} \cdot \alpha \pi_{s}\right] .
$$

We also have

$$
\alpha \pi=\sum \alpha \pi_{s}=\sum\left[A_{s+1} \alpha \pi_{s}+A_{s-1} \cdot \alpha \pi_{s}\right] .
$$

It is clearer if we write these out in full, retaining only the terms actually present,

$$
\begin{aligned}
& \alpha \pi=A_{0} \cdot \alpha \pi_{1}+A_{1} \cdot \alpha\left(\pi_{0}+\pi_{2}\right) \\
& \quad+A_{2} \cdot \alpha\left(\pi_{1}+\pi_{3}\right)+A_{3} \cdot \alpha\left(\pi_{2}+\pi_{4}\right)+\cdots, \\
& \begin{array}{r}
\alpha \alpha=A_{0} \cdot \alpha \pi_{1}+A_{1} \cdot \alpha\left(\pi_{0}-\pi_{2}\right) \\
\quad-A_{2} \cdot \alpha\left(\pi_{1}-\pi_{3}\right)+A_{3} \cdot \alpha\left(\pi_{2}-\pi_{4}\right)+\cdots .
\end{array}
\end{aligned}
$$

Of course when $\pi$ is even all the even terms are zero in these, and when $\pi$ is odd all the odd terms are zero. We have from these, by combining,

$$
\begin{aligned}
& \frac{1}{2}[\alpha \pi+\pi \alpha]=A_{0} \cdot \alpha \pi_{1}+A_{1} \cdot \alpha \pi_{0}+A_{2} \cdot \alpha \pi_{3}+A_{3} \cdot \alpha \pi_{2}+\cdots, \\
& \frac{1}{2}[\alpha \pi-\pi \alpha]=A_{1} \cdot \alpha \pi_{2}+A_{2} \cdot \alpha \pi_{1}+A_{3} \cdot \alpha \pi_{4}+A_{4} \cdot \alpha \pi_{3}+\cdots .
\end{aligned}
$$

We have a number of interesting results from these. For instance, taking $A_{0}$ of both sides we find that

$$
A_{0} \cdot \pi \alpha=A_{0} \cdot \alpha \pi .
$$

Hence $A_{0}$ is invariant under the substitution $(1,2, \cdots, n)$. This substitution taken with the other which leaves $A_{0}$ invariant gives a group of order $2 n$.

* Since $A_{0}\left(\pi_{i}-\bar{\pi}\right)=0, A_{4} \cdot(\pi-\bar{\pi})=0$, etc. 
Since in the expanded form, where $n=2 m$, we see that there are $2^{m}(m !)$ substitutions which leave the individual terms unchanged, and there are $(2 m)$ ! substitutions altogether, there will be in the development of $A_{0} \cdot \alpha_{1} \cdots \alpha_{n}$

$$
\frac{(2 m) !}{2^{m}(m !)}=(2 m-1)(2 m-3) \cdots 3 \cdot 1 \text { terms. }
$$

There will evidently be for $n=2 m$ vectors of grade $1, \frac{1}{2}(n-1) !$ different expressions $A_{0} \cdot \alpha_{1} \cdots \alpha_{n}$.

Again we have

$$
\begin{aligned}
& A_{1} \cdot \pi \alpha=A_{1} \cdot \alpha \pi-2 A_{1} \cdot \alpha \pi_{2}=-A_{1} \cdot \alpha \pi+2 \alpha \pi_{0} ; \\
& A_{2} \cdot \pi \alpha=A_{2} \cdot \alpha \pi-2 A_{2} \cdot \alpha \pi_{1}=-A_{2} \cdot \alpha \pi+2 A_{2} \cdot \alpha \pi_{3} ; \\
& A_{3} \cdot \pi \alpha=A_{3} \cdot \alpha \pi-2 A_{3} \cdot \alpha \pi_{4}=-A_{3} \cdot \alpha \pi+2 A_{3} \cdot \alpha \pi_{2} ; \\
& \cdot \cdot \cdot \cdot \cdot \cdot \cdot \cdot \cdot \cdot \cdot \cdot \cdot \cdot \cdot \cdot \cdot \cdot \cdot \cdot \cdot \cdot \cdot \cdot \cdot \\
& A_{m} \cdot \pi \alpha=A_{m} \cdot \alpha \pi-2 A_{m} \cdot \alpha \pi_{m \neq 1}=-A_{m} \cdot \alpha \pi+2 A_{m} \cdot \alpha \pi_{m \neq 1},
\end{aligned}
$$

where the upper signs are used if $m$ is odd, the lower if $m$ is even. Returning to our previous procedure we have

$$
\begin{array}{r}
\mu \alpha \beta=\mu A_{0} \cdot \alpha \beta+\mu A_{2} \cdot \alpha \beta=\mu A_{0} \cdot \alpha \beta+A_{m-2} \cdot \mu A_{2} \cdot \alpha \beta \\
\quad+A_{m} \cdot \mu A_{2} \cdot \alpha \beta+A_{m+2} \cdot \mu A_{2} \cdot \alpha \beta, \\
=\mu A_{0} \cdot \alpha \beta+A_{m-2} \cdot\left(A_{2} \cdot \alpha \beta\right) \mu \\
-A_{m} \cdot\left(A_{2} \cdot \alpha \beta\right) \mu+A_{m+2} \cdot\left(A_{2} \cdot \alpha \beta\right) \mu, \\
\alpha \beta \mu=\mu A_{0} \cdot \alpha \beta+A_{m-2} \cdot\left(A_{2} \cdot \alpha \beta\right) \mu+A_{m} \cdot\left(A_{2} \cdot \alpha \beta\right) \mu+A_{m+2} \cdot\left(A_{2} \cdot \alpha \beta\right) \mu .
\end{array}
$$

We have at once

whence easily

$$
\begin{gathered}
\mu \alpha \beta=\alpha \beta \mu-2 A_{m}\left(A_{2} \cdot \alpha \beta\right) \mu, \\
\pi \alpha \beta=\alpha \beta \pi-2 \sum A_{m}\left(A_{2} \cdot \alpha \beta\right) \pi_{m} .
\end{gathered}
$$

We need not proceed in this fashion, for it is simpler to make use of the conjugates, thus: let

then

$$
\pi=\alpha_{1} \cdots \alpha_{n} \quad \text { and } \quad \tau=\beta_{1} \cdots \beta_{p} ;
$$

and since

$$
\pi \tau=\overline{\bar{\tau} \bar{\pi}}=\sum(-)^{\frac{1}{m(m+1)}} A_{m} \cdot \bar{\tau} \bar{\pi},
$$

$$
\tau \pi=\sum A_{m} \cdot \tau \pi
$$

we have the general formula

$$
\pi \tau \pm \tau \pi=\sum A_{m} \cdot\left(\tau \pi \pm(-)^{\frac{1}{m}(m+1)} \bar{\tau} \bar{\pi}\right) .
$$

Now by expanding each expression in the form

$$
\pi=\sum \pi_{s}, \quad \bar{\pi}=\sum(-)^{\hat{l}_{s}(s+1)} \pi_{s},
$$


and similarly for $\tau$, and cancelling all terms that must give 0 , we arrive at any of these forms directly. The terms retained are of the form $\tau_{p} \pi_{s}$ where

$$
m \leqq|p-s|, \quad p+s \leqq m .
$$

If we take conjugates of the expression just written we have

$$
\pm \bar{\pi} \bar{\tau}+\bar{\tau} \bar{\pi}=\sum A_{m} \cdot\left[(-)^{\frac{1}{m(m+1)}} \tau \pi \pm \bar{\tau} \bar{\pi}\right] \text {. }
$$

By combining these formulas by addition and subtraction we arrive at a variety of forms.

Applying the same method to three products as factors of a single product we see that

$$
\tau \pi \sigma=\sum(-)^{\frac{1}{2} m(m+1)} A_{m} \cdot \bar{\sigma} \bar{\pi} \bar{\tau} .
$$

We combine this with $\sigma \pi \tau=\sum A_{m} \cdot \sigma \pi \tau$ to produce various formulas. In particular let $\sigma$ and $\tau$ be vectors of grade 1 ; then $\sigma=-\sigma$ and $\bar{\tau}=-\tau$, so that

We have then

$$
\tau \pi \sigma=\sum(-)^{\frac{1}{2 m(m+1)}} A_{m} \cdot \sigma \bar{\pi} \tau .
$$

$$
\sigma \pi \tau \pm \tau \pi \sigma=\sum A_{m} \cdot \sigma\left[\pi \pm(-)^{\frac{1}{2} m(m+1)} \bar{\pi}\right] \tau .
$$

Remembering the grades of $\sigma$ and $\tau$, the only possible grades for the [] which will not give zero terms are the grades $m-2, m, m+2$. The bracket therefore reduces to $2 \pi_{m}$ for the upper sign and $2\left(\pi_{m-2}+\pi_{m+2}\right)$ for the lower sign. Hence

$$
\begin{aligned}
& \sigma \pi \tau+\tau \pi \sigma=2 \sum A_{m} \cdot \sigma \pi_{m} \tau, \\
& \sigma \pi \tau-\tau \pi \sigma=2 \sum A_{m} \cdot\left[\sigma \pi_{m-2} \tau+\sigma \pi_{m+2} \tau\right] .
\end{aligned}
$$

We finally reduce these as follows:

$$
\begin{aligned}
\sigma \pi \tau+\tau \pi \sigma & =2 \sum A_{m} \cdot \sigma\left[A_{m+1} \cdot \pi_{m} \tau+A_{m-1} \cdot \pi_{m} \tau\right] \\
& =2 \sum(-)^{m} A_{m} \cdot \sigma\left[A_{m+1} \cdot \tau \pi_{m}-A_{m-1} \cdot \tau \pi_{m}\right] \\
& = \pm 2 \sum\left[\pi_{m} A_{0} \cdot \sigma \tau-A_{m} \cdot \sigma A_{m-1} \cdot \tau \pi_{m}\right. \\
& \left.\quad-A_{m} \cdot \tau A_{m-1} \cdot \sigma \pi_{m}\right], \\
\sigma \pi \tau-\tau \pi \sigma & = \pm 2 \sum A_{m}\left(A_{2} \cdot \sigma \tau\right)\left(\pi_{m-2}+\pi_{m+2}\right)
\end{aligned}
$$

These formulas give the effect of transposing two vectors in such a product. They are very useful For instance when we introduce $\nabla$ it will be seen that we may set $\nabla$ for $\sigma$, and for $\nabla A_{0} \cdot \tau()+\tau A_{0} \cdot \nabla()$ we may write $2 \phi_{0}()$. If then we set $\pi_{m}^{1}$ for the value of $\pi$ when in the vector of grade $m$ in each term of the expansion for $\pi_{m}$ (say $A_{m} \cdot \gamma_{1} \cdots \gamma_{m}$ ) we write $\phi_{0}$, thus:

$$
\begin{aligned}
A_{m} \cdot \phi_{0}\left(\gamma_{1}\right) \gamma_{2} \cdots \gamma_{m}+A_{m} \cdot \gamma_{1} \phi_{0}\left(\gamma_{2}\right) & \cdots \gamma_{m} \\
& +\cdots+A_{m} \cdot \gamma_{1} \gamma_{2} \cdots \phi_{0}\left(\gamma_{m}\right),
\end{aligned}
$$

we have

$$
\nabla \pi \tau+\tau \pi \nabla= \pm 2 \pi A_{0} \cdot \nabla \tau \mp 2 \sum \pi_{m}^{1}
$$


where $\nabla$ acts only on $\tau$. The expression $A_{0} \cdot \nabla \tau$ will be called later the divergence of $\tau$. Likewise $A_{2} \cdot \nabla \tau$ will be called the curl of $\tau$. We see then that when curl $\tau=0, \nabla \pi \tau=\tau \pi \nabla$. We cannot elaborate on these, but this single example will show how simple the transformations are without any great display of formulas of reduction.*

A few cases need to be written down, however. They will explain themselves. We use $\pi$ and $\tau$ as products of grades $i$ and $j$ respectively, and $\sigma$ as of grade 1:

$$
\begin{aligned}
\pi_{i} \tau_{j} & =(-)^{i j}\left[A_{i+j}-A_{i+j-2}+A_{i+j-4}-\cdots\right] \tau_{j} \pi_{i} ; \\
\frac{1}{2}\left(\pi_{i} \tau_{j}+(-)^{i j} \tau_{j} \pi_{i}\right) & =\left[A_{i+j}+A_{i+j-4}+\cdots\right] \pi_{i} \tau_{j} ; \\
\frac{1}{2}\left(\pi_{i} \tau_{j}-(-)^{i j} \tau_{j} \pi_{i}\right) & =\left[A_{i+j-2}+A_{i+j-6}+\cdots\right] \pi_{i} \tau_{j} ; \\
A_{i+j-1} \pi_{i} \sigma \tau_{j} & =A_{i+j-1}\left[\pi_{i} A_{j+1} \cdot \sigma \tau_{j}+\left(A_{i-1} \cdot \pi_{i} \sigma\right) \tau_{j}\right. \\
& \left.+(-)^{i} \sigma A_{i+j} \cdot \pi_{i} \tau_{j}\right] ; \\
A_{i-j+1} \pi_{i} \sigma \tau_{j} & =A_{i-j+1}\left[\pi_{i} A_{j-1} \cdot \sigma \tau_{j}+\left(A_{i-1} \cdot \pi_{i} \sigma\right) \tau_{j}\right. \\
& \left.+(-)^{i} \sigma A_{i+j} \cdot \pi_{i} \tau_{j}\right] .
\end{aligned}
$$

23. We consider now more particularly $A_{0} \cdot \alpha_{1} \cdots \alpha_{2 n}$. In the first place from the development it is obvious that the form is a pfaffian. Hence if we square it we shall have a skew determinant of even order, that is, $\left(A_{0} \cdot \alpha_{1} \cdots \alpha_{2 n}\right)^{2}$

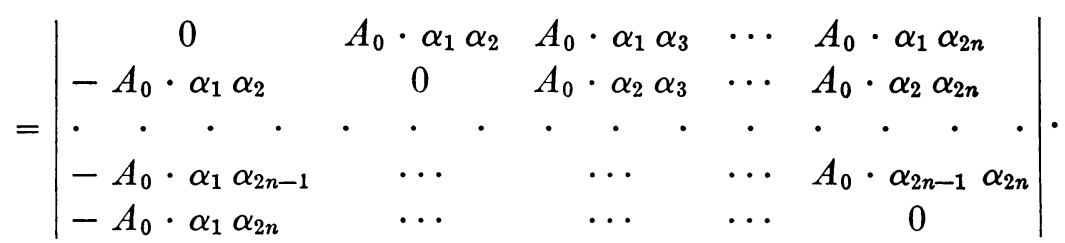

It is evident in this form that advancing each subscript cyclically is equivalent to moving the first row and column to the last row and column, which does not change the value of the determinant. Reversing all rows and columns does not change the determinant. The number of positive terms in the pfaffian is one greater than the number of negative terms, hence not all terms can have their signs changed without changing the value. This shows again the permutations possible in this form. If any vector is orthogonal to all the others the pfaffian vanishes. From the form $\sigma \pi \tau+\tau \pi \sigma$ if $m=0$ we have

$$
A_{0} \cdot \sigma \pi \tau+A_{0} \cdot \tau \pi \sigma=2 A_{0} \cdot \pi A_{0} \cdot \sigma \tau .
$$

Hence if $\sigma$ and $\tau$ are orthogonal the right side vanishes. Hence interchanging two orthogonal vectors in the $A_{0}$. form merely changes its sign.

Let $A_{0} \cdot()$ represent $A_{0} \cdot \alpha_{1} \cdots \alpha_{2 n}, A_{0} \cdot\left(i_{1}, i_{2}, \cdots, i_{2_{m}}\right)$ represent $A_{0} \cdot \alpha_{1} \ldots \ldots \ldots \alpha_{2 n}, m<n$, and let $(-)^{i+k} A_{i k} \cdot \ldots,,_{2 m}$ be the minor of $A_{0} \cdot \alpha_{i} \alpha_{k}$. It is clear that if we differentiate the expressions $A_{0} \cdot()$ as to

* By means of these forms we can reduce every expression $A_{m} \cdot \alpha_{1} \cdots \alpha_{n}$ to sums and differences of products alone, thus $A_{0} \cdot \alpha_{1} \alpha_{2}=\frac{1}{2}\left(\alpha_{1} \alpha_{2}+\alpha_{2} \alpha_{1}\right), A_{2} \cdot \alpha_{1} \alpha_{2}=\frac{1}{2}\left(\alpha_{1} \alpha_{2}-\alpha_{2} \alpha_{1}\right), A_{1} \cdot \alpha_{1} \alpha_{2} \alpha_{3}$ $=\frac{1}{2}\left(\alpha_{1} \alpha_{2} \alpha_{3}+\alpha_{3} \alpha_{2} \alpha_{1}\right)$. 
$A_{0} \cdot \alpha_{i} \alpha_{k}$ partially, we have $(-)^{i+k-1} A_{0} \cdot(i, k)$. If we differentiate the determinant in the same manner we have $2(-)^{i+k} A_{i k}$. Hence

$$
A_{i k}=-A_{0} \cdot() A_{0} \cdot(i, k) \text {. }
$$

From a well known theorem in determinants if

$$
\Delta\left(i_{1}, i_{2}, \cdots, i_{m} ; j_{1}, j_{2}, \cdots, j_{m}\right)
$$

is the determinant produced by erasing from the original determinant the rows indicated by the $i$ subscripts, and the columns indicated by the $j$ subscripts, then we have

$$
\left|A_{i_{x} y_{y}}\right|=\left[A_{0} \cdot()\right]^{2 m-2} \Delta\left(i_{1} \cdots j_{1} \cdots\right) \quad(x, y=1,2, \cdots, m) .
$$

In case the subscripts $i$ and $j$ are the same, $\Delta$ is a skew symmetric determinant also and is the square of $A_{0} \cdot\left(i_{1}, \cdots, i_{m}\right)$. The determinant on the left in any case reduces to

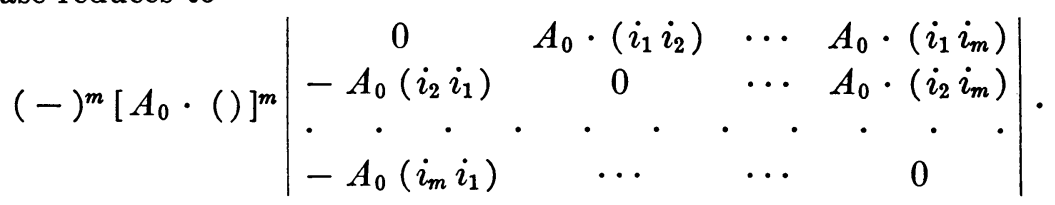

This vanishes if $m$ is odd, giving a theorem as to $\Delta$, and if $m$ is even the determinant is the square of a pfaffian, giving a series of theorems about expressions built up from the forms $A_{0} \cdot(i, j)$ in the same formal manner as the forms $A_{0}$ are built up from $A_{0} \cdot \alpha_{i} \alpha_{j}$. We cite only the following:

$$
\begin{aligned}
& A_{0} \cdot(a, b) A_{0} \cdot(c, d)-A_{0} \cdot(a, c) A_{0} \cdot(b, d) \\
& \quad+A_{0} \cdot(a, d) A_{0} \cdot(b, c)=A_{0} \cdot(a, b, c, d) \cdot A_{0} \cdot() ; \\
& A_{0} \cdot(a, b) A_{0} \cdot(c, d, e, f)-\cdots=A_{0} \cdot(a, b, c, d, e, f) A_{0} \cdot() .
\end{aligned}
$$

They are easily written and extended to all orders.

24. Space does not permit further developments here, but enough has been said to make it easy to continue. The particular forms that are related to the linear vector operator, whose general form would be

$$
\phi()=\sum \alpha A_{0} \cdot \beta(),
$$

furnish a complete part by themselves. When the number of vector terms becomes infinite we have functional transformations, and functions of lines with related subjects enter. This development is deferred to later papers.

\section{THE DIFFERENTIATOR}

25. We should however mention here the differentiator (corresponding to the Hamiltonian nabla) related to a vector $\rho=\sum x_{i} \alpha_{i}$, expressed as depending upon $n$ parameters, $i=1,2, \cdots, n$,

$$
\nabla=\sum \alpha_{i} \partial / \partial x_{i}
$$


This symbol may be used anywhere just as if it were a vector, for all vector properties, and is supposed to differentiate in situ any vector dependent upon the parameters entering $\rho$. It differentiates also in situ any function or operator dependent upon these parameters. This one simple rule enables us to write out at once an indefinitely great number of forms for differential work. No special collection of formulas is necessary. The mere fact that $\nabla$ may follow an operand is a principle that introduces great simplicity. We observe here that in the case of functions the process of differentiation of a function of a line used by Volterra is a case of the use of a $\nabla$ in which the number of parameters is nondenumerably infinite.

It is evident that we have

$$
d \cdot()=A_{0} \cdot d \rho \nabla \cdot()
$$

for any operand dependent upon the parameters of $\rho$. (Note the parameters of $\rho$ belong to the complex in which $\rho$ is variable, and do not mean the coördinates of $\rho$, which would be usually more numerous and might be infinite in number.) A simple example of the use of $\nabla$ is shown in the following.

If we have a pfaffian differential form $w=\sum y_{i} d x_{i}$ we know that it may be reduced to a set of terms $u d v+p d q+r d s+\cdots$. In case there is but one term and that is $d v$, the expression is called integrable or exact. If the single term is $u d v$ it is said to be integrable with a multiplier, $u^{-1}$. The number of such terms was shown by Pfaff to be not more than half the number of parameters $x$, if this is even, and not more than half the number plus one, if the number is odd.

Suppose we can reduce $w$ to $u d v$. Then we have by setting $\sigma=u \nabla v$

$$
\nabla \sigma=\nabla u \nabla v+u \nabla^{2} v, \quad \text { where } \quad \nabla^{2}=\nabla \nabla,
$$

whence

$$
A_{2} \cdot \nabla u \nabla v=A_{2} \cdot \nabla \sigma, \quad \text { and } \quad A_{3} \cdot \sigma \nabla \sigma=0 .
$$

This is a necessary condition of integrability with a multiplier. In case $u=1$, we have as the necessary condition $A_{2} \cdot \nabla \sigma=0$. Considering likewise the next form

$$
w=u d v+d s=A_{0} \cdot d \rho(u \nabla v+\nabla s),
$$

we set

$$
\sigma=u \nabla v+\nabla s
$$

whence

$$
\nabla \sigma=\nabla u \nabla v+u \nabla^{2} v+\nabla^{2} s,
$$

$$
A^{2} \cdot \nabla \sigma=A^{2} \cdot \nabla u \nabla v, \quad A_{3} \cdot \sigma \nabla \sigma=A_{3} \cdot \nabla s \nabla u \nabla v, \quad A_{4} \cdot \nabla \sigma \nabla \sigma=0 .
$$

This problem has been studied by Cartan* who gives the conditions above in

\footnotetext{
*Annales de l'École Normale (3), vol. 18 (1901), pp. 241-311.
} 
scalar forms which, owing to the arbitrary differentials, are equivalent to these:

$$
\begin{aligned}
A_{0} \cdot A_{2} \nabla \sigma A_{2} \cdot \delta_{1} \rho \delta_{2} \rho & =0, \\
A_{0} \cdot A_{3} \cdot \sigma \nabla \sigma A_{3} \cdot \delta_{1} \rho \delta_{2} \rho \delta_{3} \rho & =0, \quad \text { etc. }
\end{aligned}
$$

The expression $A_{0} \cdot \nabla \sigma$ is called the divergence of $\sigma$, and $A_{2} \cdot \nabla \sigma$ is called the curl of $\sigma$.

We have other differential expressions, as for instance in the ordinary relativity theory in four variables, we consider $A_{3} \cdot \nabla \sigma_{2}$ called the curl of the 2 -vector (vector of grade 2) $\sigma_{2}$. The complement or adjunct of this in the region of order 4 is called the Lorentzian of $\sigma$. It is $A_{1} \cdot A_{3} \nabla \sigma_{2} A_{4} \omega_{4}$.

We may build up expressions with $\nabla$ which are covariant for unique and invertible transformations $\phi(\rho)$. Such are

$$
A_{0} \cdot \nabla_{2} \alpha \pi A_{2} d_{1} \rho d_{2} \rho, A_{0} \cdot A_{3} \cdot \pi \nabla \pi A_{3} \cdot d_{1} \rho d_{2} \rho d_{3} \rho, \quad \cdots .
$$

In fact the possibility of expressing a function in such wise implies certain covariant properties.

26. In general if we write the form $A_{0} \cdot d \rho \sigma$, and consider the equation

$$
A_{0} \cdot d \rho \sigma=0 \text {, with } A_{2 n+1} \cdot \sigma \nabla \sigma \nabla \sigma \cdots \nabla \sigma=0 \text { identically, }
$$

we undertake to find $v$ such that $A_{2 n} \cdot \nabla v \sigma \nabla \sigma \cdots \nabla \sigma=0$, where both forms $A$ are alternants. From the latter, multiplying by $d \rho$ and taking $A_{2 n-1} \cdot$ we have

$$
\begin{aligned}
A_{2 n-1} d \rho A_{2 n} & \cdot \nabla v \sigma \nabla \sigma \cdots \nabla \sigma=0=d v A_{2 n-1} \cdot \sigma \nabla \sigma \cdots \nabla \sigma \\
& -A_{0} \cdot d \rho \sigma A_{2 n-1} \cdot \nabla v \nabla \sigma \cdots \nabla \sigma \\
& +A_{2 n-1} \nabla v \sigma d \sigma \nabla \sigma \cdots-A_{0} d \rho \sigma A_{2 n-1} \nabla v \sigma \nabla \sigma \cdots+\cdots \\
= & d v A_{2 n-1} \sigma \nabla \sigma \cdots-(n-1) A_{0} \cdot d \rho \sigma A_{2 n-1} \nabla v \nabla \sigma \\
& \cdots+(n-1) A_{2 n-1} \nabla v d \sigma \nabla \sigma \cdots+\cdots .
\end{aligned}
$$

Set $d v=0$, so that one solution is $v=$ const., and the remainder of the expression furnishes an equation which must hold for arbitrary differentials of the parameters of $\rho$. Setting their coefficients equal to 0 , we are able to find $v$. For instance let us have

$$
x_{1} x_{3} d x_{2}+x_{1} x_{2} d x_{3}+\left(x_{1}+x_{3} x_{5}\right) d x_{4}+x_{3} x_{4} d x_{5}=0 .
$$

We have $\sigma=x_{1} x_{3} \alpha_{2}+x_{1} x_{2} \alpha_{3}+\left(x_{1}+x_{3} x_{5}\right) \alpha_{4}+x_{3} x_{4} \alpha_{5}$, the vectors $\alpha_{1}, \cdots, \alpha_{5}$ being constant. It is easy to show that $A_{5} \cdot \sigma \nabla \sigma \nabla \sigma=0$ and four variables will suffice. To find $v$ so as to reduce the expression, we notice that since the form will be $u d v+r d s$

$$
\begin{gathered}
\sigma=u \nabla v+r \nabla s, \quad A_{2} \cdot \nabla \sigma=A_{2} \cdot \nabla u \nabla v+A_{2} \cdot \nabla r \nabla s, \\
A_{3} \cdot \sigma \nabla \sigma=r A_{3} \cdot \nabla s \nabla u \nabla v+u A_{3} \cdot \nabla v \nabla r \nabla s .
\end{gathered}
$$

Either $\nabla v$ or $\nabla s$ is common to both, hence $A_{4} \cdot \nabla v \sigma \nabla \sigma=0$. We assume the 
form for $v$ such that

$$
\nabla v=v_{1} \alpha_{1}+v_{2} \alpha_{2}+v_{3} \alpha_{3}+v_{4} \alpha_{4}+v_{5} \alpha_{5},
$$

and substituting all values we have a vector of grade 4 which vanishes so that each coefficient of the alternants $A_{4} \cdot \alpha_{1} \alpha_{2} \alpha_{3} \alpha_{4}, A_{4} \cdot \alpha_{1} \alpha_{2} \alpha_{3} \alpha_{5}$, etc., vanishes. These are here

$$
\begin{array}{rl}
x_{3}^{2} x_{5} v_{3}-x_{2} x_{3} x_{5} v_{2}+x_{1} x_{3} x_{4} v_{1} & =0, \\
x_{3}^{2} x_{4} v_{3}-x_{2} x_{3} x_{4} v_{2}+x_{1} x_{3} x_{4} v_{1} & =0, \\
-x_{2} x_{3} x_{5} v_{5}+x_{2} x_{3} x_{4} v_{4}-x_{3} x_{4} v_{3}+\left(x_{3} x_{4} x_{5}-x_{1}-x_{3} x_{5}\right) v_{1}=0 & 0 \\
-x_{1} x_{3} x_{5} v_{5}+x_{1} x_{3} x_{4} v_{4}+\left(x_{3} x_{4}-x_{1}-x_{3} x_{5}\right) v_{2}=0 .
\end{array}
$$

From these we have

$$
\begin{aligned}
x_{1} v_{1}-x_{2} v_{2}+x_{3} v_{3} & =0, \\
x_{4} v_{2}-x_{3} x_{4} v_{4}+x_{3} x_{5} v_{5} & =0 .
\end{aligned}
$$

These are satisfied by taking either $v=x_{1} / x_{3}$, or $x_{2} x_{3}+x_{4}$, or $x_{4} x_{5}$.

The equation $A_{0} \cdot d \rho \sigma=0$ means that $d \rho$ is everywhere orthogonal to the congruence given by $A_{2} d \rho \sigma=0$. This is called the characteristic congruence. If the equation is integrable with or without a multiplier, the congruence satisfies $A_{2} \cdot \nabla \sigma=0$ or $A_{3} \cdot \sigma \nabla \sigma=0$, and this makes it a normal congruence. That is, there is a function of $n-1$ of the parameters, $f$, such that $A_{0} \sigma \nabla f=0$. The integral in fact is $f=$ const. The other cases, $A_{4} \cdot \nabla \sigma \nabla \sigma=0$, etc., give special congruences worthy of study.

27. The integral $\int A_{0} \cdot \sigma d \rho$ depends generally upon the path of $\rho$, that is, $\rho$ is taken as an arbitrary function of a single parameter, $s, \rho=\rho(s)$, and $d \rho$ becomes $\rho^{\prime} d s$. The terminal values of $s$ furnish the limits. The integral evidently depends in general upon the form of the function $\rho(s)$. If the initial and the final value of $s$ are the same we say we have integrated around $a$ loop, and indicate the fact with the sign $\varnothing$. If we follow any path for which we have $A_{0} \cdot \sigma d \rho=0$, it is evident that the integral from any point to any other is zero, and such paths are orthogonal lines for the characteristic congruence. In case they can be collected upon spreads (functions of one or more parameters) these functions are called solutions of the pfaffian form.

If we follow a loop or closed circuit in the integration, and choose a spread of order 2 (a surface), attending as usual to singularities, we may introduce elementary areas and circuits, arriving at the generalized Stokes' Theorem,

$$
\oint A_{0} \cdot \sigma d \rho=\iint A_{0} \cdot A_{2} \nabla \sigma A_{2} \cdot d_{1} \rho d_{2} \rho,
$$

over the area. This theorem still applies in case the line-integral is not taken over a circuit, if we can draw through the terminals of the actual path orthogonal lines as mentioned above so as to complete a circuit, since the integral along the lines introduced is zero. If the curl of $\sigma$ is zero at all points of the area the loop integral evidently vanishes. 


\section{INTEGRAL INVARIANTS*}

28. Suppose that we have a general function linear in $d \rho, Q(d \rho)$. If we integrate this over a loop the form of Stokes' theorem is

$$
\Phi Q(d \rho)=\int \mathcal{S} Q^{\prime}\left(A_{1} \cdot \nabla^{\prime} A_{2} d_{1} \rho d_{2} \rho\right),
$$

where the accent shows the operand of the accented $\nabla$. This may be considered to be the difference between two distinct paths from an initial point to a terminal point chosen on the loop, the double integral being taken over the enclosed area. If the paths differ infinitesimally it becomes the variation of the integral for a variation in path. Therefore if

$$
Q^{\prime}\left(A_{1} \nabla^{\prime} A_{2}[]\right)=0 \text { identically, }
$$

the variation is zero, and the integral is called an integral invariant for the variation in path. We do not necessarily have to retain the same end points, for we may move either along any line which satisfies the equation $Q(d \rho)=0$.

When the integral is invariant it is called the integral of the exact differential form $Q(d \rho)=0$. For instance, $A_{2} \cdot \alpha d \rho$ is exact, the integral being $A_{2} \cdot \alpha \rho$. In this case we have identically $A_{2} \cdot \alpha\left(A_{1} \nabla A_{2}[]\right)=0$, since $\alpha$ is constant. The lines which satisfy $A_{2} \cdot \alpha d \rho=0$ are $\rho=s \alpha$. The path could be moved parallel to $\alpha$, leaving the integral invariant.

29. It happens sometimes that an integral remains invariant when the whole loop is displaced, as for instance the strength of a vortex tube. The general condition is obtained thus: let the integral be taken over a region of order $n$ (that is, there are $n$ independent parameters and these are varied between appropriate limits),

$$
I_{n}=\mathcal{S}^{n} Q\left(A_{n} \cdot d_{1} \rho d_{2} \rho \cdots d_{n} \rho\right),
$$

where $\boldsymbol{S}^{n}$ means $n$ integral signs or an $n$-fold integral (the iteration of an integral in order to procure an area-integral, or a space-integral, is incidental here and may not be necessary). Suppose now that we displace the region so that $\rho$ becomes $\rho+\delta \rho$; then we may consider the effect of this on any function of $\rho$ by noting that

Hence

$$
\delta=A_{0} \cdot \delta \rho \nabla
$$

$$
\begin{aligned}
& \delta I_{n}=A_{0} \cdot \delta \rho \nabla \mathcal{S}^{n} Q\left(A_{n} \cdot \delta_{1} \rho \cdots d_{n} \rho\right) \\
& =\mathcal{S}^{n} A_{0} \cdot \delta \rho \nabla^{\prime} \cdot Q^{\prime}\left(A_{n} \cdots\right)+\mathcal{S}^{n} Q\left(A_{n} d_{1} \delta \rho d_{2} \rho \cdots d_{n} \rho\right) \\
& +\cdots+\int^{n} Q\left(A_{n} \cdot d_{1} \rho \cdots d_{n} \delta \rho\right) \\
& =\int^{n} A_{0} \delta \rho \nabla^{\prime} \cdot Q^{\prime}\left(A_{n} \cdot d_{1} \rho \cdots d_{n} \rho\right) \\
& +\int^{n} Q\left(A_{n} d \rho^{\prime} A_{n-1} \cdot \nabla^{\prime} A_{n} \cdot d_{1} \rho \cdots d_{n} \rho\right) .
\end{aligned}
$$

* The contents of this division were included in a paper presented before the Society, Chicago Section, January 2, 1913, Integral invariants in general vector analysis. 
This is called the first form. Integrate the last term by parts, combine, and we have

$$
\begin{aligned}
\delta I_{n}=\mathcal{S}^{n} Q^{\prime}\left(A_{n} \cdot \nabla^{\prime} A_{n+1} \delta \rho\right. & \left.d_{1} \rho \cdots d_{n} \rho\right) \\
& +\mathcal{S}^{n}\left[Q\left(A_{n} \cdot \delta \rho\right)\right]^{\prime} A_{n-1} \cdot \nabla^{\prime} A_{n} \cdot d_{1} \rho \cdots d_{n} \rho .
\end{aligned}
$$

This is called the second form. The expansion of the last term gives a number of terms each of which is integrable once, and we have

$$
\begin{aligned}
\delta I_{n}=\mathcal{S}^{n} Q^{\prime}( & \left.A_{n} \cdot \nabla^{\prime} A_{n+1} \cdot \delta \rho d_{1} \rho \cdots d_{n} \rho\right) \\
& +\sum \int^{n-1}\left[\left(Q A_{n} \cdot \delta \rho\right)_{B_{i}}-\left(Q A_{n} \cdot \delta \rho\right)_{A_{i}}\right] A_{n-1} \cdot d_{1} \rho \cdots d_{n} \rho .
\end{aligned}
$$

Let $\delta \rho$ follow a path so that $\delta \rho=\sigma \delta t$; then we have

$$
\begin{aligned}
\delta I_{n} / \delta t=\mathcal{S}^{n} Q^{\prime} A_{n} \cdot \nabla^{\prime} A_{n+1} & \cdot \sigma d_{1} \rho \cdots d_{n} \rho \\
& +\sum \int^{n-1}\left[\left(Q A_{n} \cdot \sigma\right)_{B_{i}}-\left(Q A_{n} \cdot \sigma\right)_{A_{i}}\right] A_{n-1} \cdots .
\end{aligned}
$$

If we had retained the first form we would have

$$
\delta I_{n} / \delta t=\mathcal{S}^{n} A_{0} \cdot \sigma \nabla^{\prime} \cdot Q^{\prime}\left(A_{n} \cdots\right)+\mathcal{S}^{n} Q\left(A_{n} \cdot \sigma A_{n-1} \nabla^{\prime} A_{n} \cdots\right) .
$$

If now $I_{n}$ is an absolute invariant, we must have for the paths given by $\sigma$

$$
A_{0} \cdot \sigma \nabla^{\prime} \cdot Q^{\prime}\left(A_{n} \cdots\right)+Q\left(A_{n} \cdot \sigma^{\prime} A_{n-1} \cdot \nabla^{\prime} A_{n} \cdots\right)=0 \text { identically, }
$$

or for the second form

$$
Q^{\prime}\left(A_{n} \cdot \nabla^{\prime} A_{n+1} \cdot \sigma A_{n} \cdots\right)+\left(Q A_{n} \cdot \sigma\right)^{\prime} A_{n-1} \cdot \nabla^{\prime} A_{n} \cdots=0 \text { identically. }
$$

These conditions are very general and include every form as usually stated. As an instance, consider the integral $\int A_{0} \cdot \tau d \rho$. This will be an absolute invariant for the congruence $\sigma$ if

$$
A_{0} \cdot \sigma \nabla A_{0} \cdot \tau()+A_{0} \cdot \tau \sigma^{\prime} A_{0} \cdot \nabla^{\prime}()=0 .
$$

The condition may also be written

$$
\nabla^{\prime} A_{0} \cdot \sigma^{\prime} \tau=-\tau^{\prime} A_{0} \nabla^{\prime} \sigma \quad \text { or } \quad \nabla\left(A_{0} \cdot \sigma \tau\right)=-A_{1} \cdot \sigma A_{2} \cdot \nabla \tau .
$$

In case the boundary is fixed, then at the boundary $\delta \rho=0$; if the integral is to remain invariant for all $\sigma$, we see from the second form that as the second integral is now zero, and the first holds for all $\sigma$, we must have

$$
Q^{\prime}\left(A_{n} \cdot \nabla^{\prime} A_{n+1} \cdots\right)=0 \text { identically. }
$$

For instance, in the example just considered we would have

whence

$$
A_{0} \cdot \tau^{\prime} A_{1} \cdot \nabla^{\prime} A_{2} \cdots=0 \text { identically, }
$$

$$
A_{2} \cdot \nabla \tau=0 \text { identically. }
$$

The situation considered here is the extension from an integral over two distinct paths, with fixed terminals, to an integral over two regions of order $n$, with fixed boundary. Such an integral is indicated by Goursat* by $I_{n}^{d}$. When

\footnotetext{
*Journal des Mathématiques (6), vol. 4 (1908), pp. 331.
} 
the boundary is closed we have simply an invariant, called a relative invariant, indicated by $J_{n}$.

3c. In a manner similar to that for Stokes' generalized theorem we have Green's generalized theorem

$$
\begin{aligned}
I_{n}=I_{n+1}^{a}=\mathscr{S}^{n} Q\left(A_{n} \cdot d_{1} \rho \cdots\right. & \left.d_{n} \rho\right) \\
& =\int^{n+1} Q^{\prime}\left(A_{n} \cdot \nabla^{\prime} \cdot A_{n+1} \cdot d_{1} \rho \cdots d_{n+1} \rho\right) .
\end{aligned}
$$

The second integral is evidently of the type $I^{d}$. Since now a relative invariant integral may be written as an integral of order higher by unity, the condition of its being invariant may be stated. That is,

$$
J_{n}=\oint^{n} Q\left(A_{n} \cdot d_{1} \rho \cdots d_{n} \rho\right)
$$

is a relative invariant if

$$
I_{n+1}^{d}=\int^{n+1} Q^{\prime}\left(A_{n} \nabla^{\prime} A_{n+1} \cdot d_{1} \rho \cdots d_{n+1} \rho\right)
$$

is absolute, that is to say, if or if

$$
A_{0} \cdot \sigma \nabla^{\prime} \cdot Q^{\prime}\left(A_{n} \cdot \nabla^{\prime} A_{n+1}\right)+Q^{\prime}\left(A_{n} \nabla^{\prime} A_{n+1} \sigma^{\prime \prime} A_{n} \nabla^{\prime \prime} A_{n+1}\right)=0,
$$

$$
Q^{\prime}\left(A_{n} \cdot \nabla^{\prime} A_{n+1} \nabla^{\prime} A_{n+2} \cdot \sigma \cdots\right)+\left(Q^{\prime} A_{n} \cdot \nabla^{\prime} A_{n+1} \cdot \sigma\right)^{\prime \prime} A_{n} \cdot \nabla^{\prime \prime} A_{n+1}=0 .
$$

This gives us the condition (since $A_{n} \cdot A_{2} \nabla^{\prime} \nabla^{\prime} A_{n+2} \cdots$ vanishes identically)

$$
\left[Q^{\prime} A_{n} \cdot \nabla^{\prime} A_{n+1} \cdot \sigma\right]^{\prime \prime} A_{n} \cdot \nabla^{\prime \prime} A_{n+1} \cdots=0 \text {. }
$$

For instance in the case considered above

$$
A_{0} \cdot \tau^{\prime} A_{1} \cdot \nabla^{\prime} A_{2} \cdot \sigma^{\prime \prime} A_{1} \cdot \nabla^{\prime \prime} A_{2} \cdot()+A_{0} \cdot \tau^{\prime} A_{1} \cdot \nabla^{\prime} A_{2} \cdot \nabla^{\prime} A_{3} \cdot \sigma A_{2}()=0 \text {. }
$$

Hence, reducing, $A_{2} \cdot \nabla A_{1} \cdot \sigma A_{2} \cdot \nabla \tau=0$ identically.

31. Since we may always write

$$
\begin{aligned}
A_{0} \cdot \sigma \nabla^{\prime}\left(Q^{\prime} A_{n} \cdot \sigma \cdots\right) & +Q A_{n} \cdot \sigma A_{n-1} \sigma^{\prime} A_{n-2} \nabla^{\prime} A_{n-1} \\
& =A_{0} \cdot \sigma \nabla^{\prime} \cdot Q^{\prime}\left(A_{n} \cdot \sigma \cdots\right)+Q\left(A_{n} \cdot \sigma^{\prime} A_{n-1} \nabla^{\prime} A_{n} \sigma\right),
\end{aligned}
$$

the condition above becomes

$$
A_{0} \cdot \sigma \nabla^{\prime} Q^{\prime}\left(A_{n} \cdot \sigma A_{n-1}()\right)+Q\left(A_{n} \cdot \sigma^{\prime} A_{n-1} \nabla^{\prime} A_{n} \cdot \sigma A_{n-1}\right)=0 .
$$

Hence if $I_{n}=\int^{n} Q A_{n}$ is an absolute invariant,

$$
I_{n-1}=\int^{n-1} Q\left(A_{n} \cdot \sigma A_{n-1} \cdots\right)
$$

is an absolute invariant.

32. From any integrand $Q\left(A_{n} \cdots\right)$ may be formed by two processes two other integrands, one by the process $D$, giving $Q^{\prime}\left(A_{n} \cdot \nabla^{\prime} A_{n+1} \cdots\right)$, and the other by the process $E$, giving $Q\left(A_{n} \cdot \sigma A_{n-1} \cdots\right)$. In case the latter form 
vanishes identically, we mark the integral $I_{n}$ of $Q\left(A_{n} \cdots\right)$ with an $e$, thus $I_{n}^{e}$. In any case the second application of the $E$ process gives an identically vanishing form, hence from any $I_{n}$ the $E$ process produces an $I_{n-1}^{e}$. Integrals are of four types with respect to these processes, that is, according to whether or not they can be marked with $d$ or $e$. The effect of the $D$ process on the integrand is to mark the integral with $d$, of the $E$ process to mark the integral with $e$. We need to prove that if an integral invariant has either character the other process will not destroy this character. That is,

$$
\left(I^{d}\right)^{e}=\left(I^{e}\right)^{d} .
$$

$E$ produces from $I_{n}^{d}$ an $I_{n-1}^{d e}$, that is, if the first is from an exact integrand, the second will also be exact when the first is invariant, and also will be invariant. For from

$$
Q^{\prime}\left(A_{n} \nabla^{\prime} \cdots\right)=0
$$

and

$$
Q^{\prime}\left(A_{n} \cdot \nabla^{\prime} A_{n+1} \cdot \sigma \cdots\right)+\left(Q A_{n} \cdot \sigma \cdots\right)^{\prime} A_{n-1} \nabla^{\prime} A_{n} \cdots=0
$$

it follows that

$$
\left(Q A_{n} \cdot \sigma\right)^{\prime} A_{n-1} \cdot \nabla^{\prime} \cdots=0,
$$

which is the condition that the result have an exact integrand. To be invariant we must also have

$$
\left(Q A_{n} \cdot \sigma A_{n-1} \sigma\right)^{\prime} A_{n-2} \cdot \nabla^{\prime} \cdots=0 \text {, }
$$

which is evident. Again when $Q A_{n} \cdot \sigma \cdots=0$ and $I_{n}$ is invariant we have also

$$
Q^{\prime} A_{n} \cdot \nabla^{\prime} A_{n+1} \cdot \sigma \cdots=0,
$$

showing at once that $Q^{\prime} A_{n} \cdot \nabla^{\prime} \cdots$ is of type $E$. That it is also invariant demands that

$$
Q^{\prime} A_{n} \nabla^{\prime} A_{n+1} \nabla^{\prime} A_{n+2} \sigma \cdots+\left(Q^{\prime} A_{n} \cdot \nabla^{\prime} A_{n+1} \cdot \sigma\right)^{\prime} A_{n+1} \nabla^{\prime} A_{n}=0,
$$

which is evident. Hence the two operations are permutable when applied to an invariant, one giving $Q^{\prime} \cdot A_{n} \nabla^{\prime} A_{n+1} \cdot \sigma A_{n} \cdots$, the other

$$
\left(Q A_{n} \sigma\right)^{\prime} A_{n-1} \nabla^{\prime} A_{n} \cdots \text {. }
$$

If $Q$ is invariant, however, the first expression is the negative of the other. The characters are then unchanged. If we start with an invariant and apply the $E$ process we arrive at an $I^{e}$ of one lower order, usually not identically zero, and if we then apply the $D$ proce $;$ we have an integral of character $I^{\text {de }}$ of the original order. For instan 'e, from $A_{0} \cdot \tau d \rho$ we have, by $D$,

$$
-A_{0} \cdot A_{2} \nabla \tau A_{2} \cdot d_{1} \rho d_{2} \rho \text {, }
$$


by $E, A_{0} \tau \sigma$. Using the other process in either case we have from the first $-A_{0} \cdot A_{2} \cdot \nabla \tau A_{2} \cdot \sigma d \rho$, from the second $\left(A_{0} \cdot \sigma \tau\right)^{\prime} A_{0} \cdot \nabla^{\prime} d \rho$. In case now $A_{0} \cdot \tau d \rho$ is an invariant the new forms are also invariant.

The $D$ process is a sort of generalization of getting the curl, while the $E$ process fixes certain paths for integration, along which the lower integral may be displaced.

As instances, let $I_{1}=\int A_{0} \cdot \tau d \rho$, and let $I$ have both characters $d, e$; that means

$$
A_{2} \cdot \nabla \tau=0,
$$

and for a certain congruence given by $d \rho=\sigma d t$,

$$
A_{0} \cdot \tau \sigma=0 \text {. }
$$

The expression is exact on account of character $d$; hence the integral is

$$
u=\text { const. }
$$

This is a function of $\rho$, and such that $\tau=z \nabla u$. Hence we have also $\nabla u$ everywhere orthogonal to the congruence line $\sigma$, by the second condition. That is, $\sigma$ gives lines lying in the spread $u=c$.

Let

then

$$
I_{2}^{d e}=\iint A_{2} \cdot \pi_{2} A_{2} \cdot d_{1} \rho d_{2} \rho
$$

$$
A_{0} \cdot A_{2} \cdot \pi_{2} A_{2} \cdot \nabla A_{3}=0, \quad A_{0} \cdot A_{2} \cdot \pi_{2} A_{2} \sigma A_{1} \cdot=0 \text { identically. }
$$

We have easily $A_{3} \cdot \nabla \pi_{2}=0, A_{0} \cdot A_{2} \cdot \pi_{2} A_{2} \sigma \lambda=0$ for every $\lambda$; that is,

$$
A_{1} \cdot \sigma A_{2} \cdot \pi_{2}=0 \text { identically. }
$$

From the first condition

hence

$$
\pi_{2}=A_{2} \nabla \xi
$$

$$
A_{1} \cdot \sigma A_{2} \nabla \xi=0 \text {. }
$$

Put $d \rho$ for $\sigma d t$, and we have along the congruence

$$
A_{1} \cdot d \rho A_{2} \nabla \xi=0, \quad \text { or } \quad d \xi=\nabla^{\prime} A_{0} \xi^{\prime} d \rho .
$$

This means, if we set $\phi$ for the linear vector function $\nabla A \cdot \xi()$,

$$
d \xi=\phi d \rho \text {. }
$$

We have then formally $d \rho=\phi^{-1} d \xi$. The complete discussion is long.

A comparison of the methods used here and those given in the coördinate treatments of these problems will show the enormous gain in simplicity.

33. To solve the differential equation

$$
Q \cdot A_{n} \cdot d \rho \pi_{n-1}=0
$$


means that we must find a congruence of curves such that if $d \rho$ is taken along any curve the equation will be satisfied, and that $x(\rho) Q$ is an absolute invariant for the curves. That is, setting $d \rho=\sigma d t$,

$$
\begin{aligned}
Q \cdot A_{n} \cdot \sigma \cdots & =0, \\
(x Q)^{\prime} A_{n} \cdot \nabla A_{n+1} \sigma \cdots+\left(x Q A_{n}\right. & \cdot \sigma)^{\prime} A_{n-1} \cdot \nabla^{\prime} A_{n} \cdots=0 \\
& =(x Q)^{\prime} A_{n} \cdot \nabla^{\prime} A_{n+1} \cdot \sigma \cdots, \\
0 & =Q^{\prime} A_{n} \cdot \nabla^{\prime} A_{n+1} \cdot \sigma+A_{0} \cdot \sigma \nabla \log x \cdot Q A_{n} .
\end{aligned}
$$

Hence we must have

$$
Q A_{n} \cdot \sigma \cdots=0, \quad Q^{\prime} A_{n} \cdot \nabla^{\prime} A_{n+1} \cdot \sigma \cdots=t Q A_{n} \cdots,
$$

for some $t$, and $t$ may be zero. For instance let $Q$ be $A_{0} \cdot \xi d \rho=0$. The condition is then

$$
A_{0} \cdot \sigma \xi=0, \quad A_{0} \cdot A_{2} \nabla \xi A_{2} \cdot \sigma()=t A_{0} \cdot \xi() .
$$

For a particular case let $\xi=A_{1} \cdot \rho A_{2} \cdot \alpha \rho$, where $\alpha$ is constant. Then $A_{2} \cdot \nabla \xi=3 A_{2} \cdot \alpha \rho, A_{0} \cdot A_{2} \cdot \sigma \rho A_{2} \cdot \alpha \rho=0, A_{1} \cdot \sigma A_{2} \cdot \alpha \rho=t A_{1} \cdot \rho A_{2} \cdot \alpha \rho$. These are satisfied if we take

$$
\sigma=t \rho+\text { any vector orthogonal to } \alpha \text { and to } \rho \text {. }
$$

Hence $d \rho$ must be in the plane through the origin containing the common perpendicular to $\alpha$ and $\rho$. Hence $d \rho$ is in any plane tangent to a cone of revolution about $\alpha$ as an axis. This cone must therefore furnish an integral, that is,

$$
A_{0} \cdot \rho \rho=c^{2}\left(A_{0} \cdot \alpha \rho\right)^{2} .
$$

34. A system of equations

$$
0=A_{0} \cdot \xi_{1} d \rho=A_{0} \cdot \xi_{2} d \rho=\cdots=A_{0} \cdot \xi_{n} d \rho_{n}
$$

is equivalent to a single equation

$$
A_{n-1} \cdot d \rho A_{n} \cdot \xi_{1} \cdots \xi_{n}=A_{n-1} \pi_{n} d \rho=0 .
$$

The conditions then reduce to

$$
A_{n-1} \cdot \pi_{n} \sigma=0, \quad A_{0} \cdot \sigma \nabla A_{n-1}() \pi_{n}-A_{0} \cdot() \nabla A_{n-1} \sigma \pi_{n}=t A_{n-1}() \pi_{n} .
$$

We cannot enlarge on the use of these forms in the study of differential equations, but enough has been said to indicate the procedures. We will, however, show the application to differential geometry.

\section{DIFFERENTIAL GEOMETRY}

1. Let $\rho$ be a vector dependent upon $n$ parameters $u_{1}, u_{2}, \cdots, u_{n}$, thus defining a region of order $n$. For instance if $n=1$ we would call the region 
a curve, if $n=2$, a surface, etc. We have then

$$
\rho=\rho\left(u_{1}, u_{2}, \cdots, u_{n}\right) .
$$

2. If now we let the parameters vary and assume that the function $\rho$ is differentiable as to each parameter, indicating the derivative by a corresponding subscript, we have

$$
d \rho=\rho_{1} d u_{1}+\rho_{2} d u_{2}+\cdots+\rho_{n} d u_{n}
$$

where $\rho_{i}=\partial \rho / \partial u_{i}$, and the $n$ vectors are in general linearly independent.

We will indicate the adjunct of $\rho_{i}$ in the region by $\rho^{i}$ so that

$$
\begin{array}{r}
\rho^{i}=(-)^{i-1} A_{1} \cdot A_{n-1} \cdot \rho_{1} \rho_{2} \cdots \rho_{i-1} \rho_{i+1} \cdots \rho_{n} A_{n} \cdot \rho_{1} \rho_{2} \\
\cdots \rho_{n} / A_{0} \cdot A_{n} \cdot \rho_{1} \cdots \rho_{n} A_{n} \cdot \rho_{1} \cdots \rho_{n} .
\end{array}
$$

For convenience we shall write $\nu=A_{n} \rho_{1} \cdots \rho_{n}$ so that $\nu$ is an alternate vector of grade $n$, which is also a function of the point at which it is calculated. We also set $(I \cdot \nu)^{2}=A_{0} \cdot \nu \nu$, and $U \nu=\nu / I \nu$ is a unit vector. Where $\nu=0$, we have singular points.

It is clear that

$$
A_{0} \cdot \rho_{i} \rho^{j}=0, \quad \text { if } \quad i \neq j, \quad \text { and } \quad A_{0} \cdot \rho_{i} \rho^{i}=1 .
$$

Further

$$
A_{n} \cdot \rho^{1} \rho^{2} \cdots \rho^{n}=\nu / A_{0} \nu \nu,
$$

which is seen by expanding each in terms of $\rho_{1}, \rho_{2}, \cdots, \rho_{n}$ and applying the theorems of determinants.

3. The expressions $A_{0} \cdot \rho_{i} \rho_{j}$ for $i, j=1, \cdots, n$ are called the fundamental quantities of the first order. They are commonly represented by $a_{i j}$. The corresponding forms $A_{0} \cdot \rho^{i} \rho^{j}=a^{i j}$ are the adjunct quantities of first order.

It is clear that

$$
\left|a_{i j}\right|=(-)^{\frac{1}{2 n(n-1)}} A_{0} \cdot \nu \nu, \quad\left|a^{i j}\right|=(-1)^{\frac{\ln (n-1)}{3} A_{0} \cdot \nu \nu .}
$$

4. The biorthogonal systems $\rho_{i}$ and $\rho^{i}$ are useful. Suppose for instance we have a system of values $l_{s}$ which we attach to $\rho_{s}$ giving a vector

$$
\lambda=\sum \rho_{s} l_{s}
$$

then we may define a new system of values

$$
m=A_{0} \cdot \rho^{r} \lambda,
$$

and from the properties of the vectors $\rho$ we have

$$
\mu=\sum m_{r} \rho_{r}=\sum \rho_{r} A_{0} \rho^{r} \lambda=\lambda .
$$

The coefficients $m$ and the coefficients $l$ therefore give the same vector when attached to the proper vectors. For the vector $\rho$ in terms of $\rho_{i}$, and the vector 
$\rho$ in terms of $\rho^{i}$ we may introduce a new system of $n$ independent vectors defining the region, and their biorthogonal system. As we are dealing with the vectors themselves we shall not be concerned with their mode of expression. Such questions however play an important part in the developments of Ricci (Lezioni sulla Teoria delle Superficie) and those who follow him.

5. If we have a vector $\xi=\sum X_{r} \rho^{r}$, and in the other form, $\xi=\sum X^{r} \rho_{r}$, then $\xi / I \rho_{r}$ is such that $X_{r} / I \rho_{r}=$ the orthogonal projection of $\xi$ on $\rho_{r}$. In other words the orthogonal projections are given by $A_{0} U \rho_{r} \xi$. Similarly for the other form.

6. If we differentiate $A_{0} \rho_{r} \rho^{8}=0$ or 1 we have in either case

$$
A_{0} \rho_{r t} \rho^{8}+A_{0} \rho_{r}\left(\rho^{8}\right)_{t}=0,
$$

a useful formula. From it we have the Christoffel * form

$$
\left\{\begin{array}{c}
r s \\
t
\end{array}\right\}=A_{0} \rho_{r s} \rho^{t}=-A_{0} \rho_{r}\left(\rho^{t}\right)_{s}=-A_{0} \rho_{s}\left(\rho^{t}\right)_{r}
$$

Evidently

$$
\begin{aligned}
\rho_{r s}=-\sum \rho_{t} A_{0} \cdot \rho_{r}\left(\rho^{t}\right)_{s} & +A_{1} \cdot U \nu A_{n+1} \cdot U \nu \rho_{r s} \\
& =-\sum \rho_{t} A_{0} \cdot \rho_{s}\left(\rho^{t}\right)_{r}+A_{1} \cdot U \nu A_{n+1} \cdot U_{\nu \rho_{r s}} \cdot
\end{aligned}
$$

The second term $\dagger$ in each form gives that part of $\rho_{r s}$ outside the region of order $n$. The other Christoffel form is

$$
\left[\begin{array}{c}
r s \\
t
\end{array}\right]=A_{0} \cdot \rho_{r s} \rho_{t}
$$

7. Since $A_{n+1} \cdot \rho_{1} \rho_{2} \cdots \rho_{n} \rho^{m}=0$ we have

$$
d A_{n+1} \cdot \nu \rho^{m}=0, \quad \text { or } \quad A_{n+1} \cdot \nu\left(\rho^{m}\right)_{z}=-A_{n+1} \cdot \nu_{z} \rho^{m},
$$

and likewise

$$
A_{n+1} \cdot \nu \rho_{r s}=-A_{n+1} \cdot \nu_{r} \rho_{s}=-A_{n+1} \cdot \nu_{s} \rho_{r} .
$$

There is usually a non-vanishing expression of first degree which is orthogonal to the region under consideration $\left(\rho_{1}, \cdots, \rho_{n}\right)$

$$
A_{1} \cdot \nu A_{n+1} \cdot \nu \rho_{r s}=-A_{1} \cdot \nu A_{n+1} \cdot \nu_{s} \rho_{r}=-A_{1} \cdot \nu A_{n+1} \cdot \nu_{r} \rho_{s} .
$$

We note that $U_{\nu}$ might as well have been used here as $\nu$. We can construct $n^{4}$ expressions

$$
A_{0} \cdot A_{n+1} \cdot \rho_{r s} \nu A_{n+1} \nu \rho_{t u},
$$

which can also be written

$A_{0} \cdot \rho_{r} \nu_{s} A_{n+1} \cdot \nu_{t} \rho_{u}$, or $A_{0} \cdot \rho_{r} A_{1} \cdot \nu_{s} A_{n+1} \cdot \nu_{t} \rho_{u}$, or $A_{0} \cdot \rho_{u} \nu_{t} A_{n+1} \cdot \rho_{r} \nu_{s}$,

* This is easily reduced to the usual cartesian form by writing out $\rho_{r s}$ in terms of $\rho_{1}, \cdots, \rho_{n}$. For the other Christoffel form expand $\rho_{r s}$ in terms of $\rho^{\prime}, \cdots, \rho^{n}$.

$\dagger$ It is to be noted that in general $\rho_{r e}$ has components not linearly expressible in terms of $\rho_{1}, \cdots, \rho_{n}$, as for instance for a surface, which is imbedded in a three-dimensional space. 
and in other forms produced by permissible interchanges of the subscripts. However this is a quadratic in $\rho_{r}, \rho_{u}$ of the form $A_{0} \rho_{r} \phi \rho_{u}$, where $\phi$ is a linear vector function. Its transverse would be

We will set

$$
A_{0} \cdot \rho_{r} \nu_{t} A_{n+1} \nu_{s} \rho_{u} \text {. }
$$

$$
\begin{aligned}
& A_{1} \cdot\left(U_{\nu}\right)_{s} A_{n+1} \cdot(U \nu)_{t}()=\mathrm{N}_{s t}(), \\
& A_{1} \cdot(U \nu)_{t} A_{n+1} \cdot(U \nu)_{s}()=\breve{\mathrm{N}}_{s t}=\mathrm{N}_{t s} .
\end{aligned}
$$

The difference of these two forms has received a symbol due to Riemann:

$$
\begin{aligned}
(r u, s t)=A_{0} \cdot \rho_{r}\left[(U \nu)_{s} A_{n+1}\right. & \cdot(U \nu)_{t} \\
& \left.-(U \nu)_{t} A_{n+1} \cdot(U \nu)_{s}\right] \rho_{u}=A_{0} \cdot \rho_{r}\left(\epsilon \mathrm{N}_{s t}\right) \rho_{u} .
\end{aligned}
$$

There is also another Riemann symbol * of order four,

$$
\{r u, s t\}=A_{0} \cdot \rho_{r} \mathrm{~N}_{s t} \rho^{u}-A_{0} \cdot \rho_{r} \mathrm{~N}_{t s} \rho^{u}=A_{0} \rho_{r}\left(\epsilon \mathrm{N}_{s t}\right) \rho^{u} \text {, }
$$

where $\epsilon(N)=\frac{1}{2}(N-\breve{N}), \breve{N}$ being the transverse linear vector function of $N$. A better form may be given these by utilizing a previous formula, $\uparrow$ which gives

$$
\begin{gathered}
\{r u, s t\}=A_{0} A_{2} \rho_{r} \rho_{u} A_{2} \cdot(U \nu)_{s}\left(U_{\nu}\right)_{t} \\
=-A_{0} \cdot \rho_{r s} U_{\nu} A_{0} \cdot \rho_{u t} U_{\nu}+A_{0} \cdot \rho_{r t} U_{\nu} A_{0} \cdot \rho_{u s} U_{\nu} \\
\{r u, s t\}=A_{0} A_{2} \rho_{r} \rho^{u} A_{2} \cdot\left(U_{\nu}\right)_{s}\left(U_{\nu}\right)_{t} \\
=-A_{0} \cdot \rho_{r s} U_{\nu} A_{0} \cdot\left(\rho^{u}\right)_{t} U_{\nu}+A_{0} \cdot \rho_{r t} U_{\nu} A_{0} \cdot\left(\rho^{u}\right)_{s} U_{\nu} .
\end{gathered}
$$

From these expressions it is evident that the two symbols in each couple may be interchanged by changing the sign, and the two couples may be interchanged.

8. The expression $A_{0} \cdot d \rho d \rho=\sum A_{0} \cdot \rho_{r} \rho_{s} d u_{r} d u_{s}$ is called the first fundamental form. In case the Riemann symbols vanish it can be reduced to the sum of not more than $n$ squares, that is, new variables may be found in terms of which the parameters $u$ may be expressed so that, for the new derived vectors $\rho^{\prime}$, we will have

$$
A_{0} \cdot d \rho d \rho=\sum A_{0} \rho_{r}^{\prime} \rho_{r}^{\prime}\left(d u_{r}^{\prime}\right)^{2} .
$$

The condition is both necessary and sufficient. If some or all of the Riemann symbols do not vanish, then it will be necessary to express $A_{0} \cdot d \rho d \rho$ as the sum of not more than $\frac{1}{2} n(n+1)$ squares. From one point of view this is equivalent to considering the region to be embedded in a region of not more than $\frac{1}{2} n(n+1)$ dimensions in which we may take the directions of $\rho_{i}$ as orthogonal.

For instance a curve on an ordinary surface has a normal in the surface and

* These may be identified with the forms commonly used by substituting and reducing.

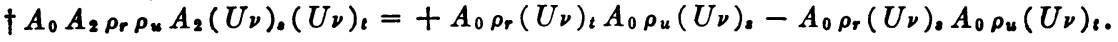


also has the normal to the surface as a normal, or indeed all lines in the plane of these two. Hence the $d s^{2}$ is expressible in terms of three differentials in ordinary space which encloses the surface, in the form

$$
d s^{2}=d x^{2}+d y^{2}+d z^{2} .
$$

On the surface alone it would usually have terms of the form

$$
d u^{2}, d u d v, d v^{2}
$$

and would not be reducible to the sum of two squares.

9. We may improve the forms we have reached so far by the introduction of the operator which generalizes the Hamilton $\nabla$. On account of this generalization we shall use $\nabla$ in a general sense. It is defined thus:

$$
\nabla=\sum \rho^{i} \partial / \partial u_{i} .
$$

It follows that $d()=A_{0} d \rho \nabla \cdot$ () for any function of the parameters $u$ only. Hence we will have $\partial / \partial u_{r}=A_{0} \cdot \rho_{r} \nabla \cdot$; therefore we may write for $(U \nu)_{r}$

The operator

$$
(U \nu)_{r}=A_{0} \rho_{r} \nabla \cdot U \nu \text {. }
$$

$$
A_{0}() \nabla \cdot U \nu=\Phi
$$

plays a very important part in all these problems and gives of course the vector rate of change of $U_{\nu}$ in the direction given by the unit vector operated upon. With this symbol we may write the Riemann symbols in the forms

$$
(r u, s t)=A_{0} A_{2} \rho_{r} \rho_{u} A_{2} \Phi \rho_{s} \Phi \rho_{t}
$$

with the understanding that $s, t$ may be interchanged with $r, u$;

$$
\{r u, s t\}=A_{0} A_{2} \rho_{r} \rho^{u} A_{2} \Phi \rho_{s} \Phi \rho_{t} .
$$

The number of Riemann symbols with two pairs of identical subscripts is $\frac{1}{2} n(n-1)$, with one pair of similar indices $\frac{1}{2}(n-1)(n-2)$, and with all different $\frac{1}{12} n(n-1)(n-2)(n-3)$, giving a total of $\frac{1}{12} n^{2}\left(n^{2}-1\right)$. It is clear that for any four directions in the region we may have a symbol $A_{0} A_{2} \alpha \beta A_{2} \Phi \gamma \Phi \delta$, which for unit vectors may be called the Riemann symbol for those directions. There is also, if we take differentials for the intensities of the vectors, a differential quadrilinear expression

$$
G_{4}=A_{0} \cdot A_{2} \cdot d^{\prime} \rho d^{\prime \prime} \rho A_{2} \cdot \Phi d^{\prime \prime \prime} \rho \Phi d^{\prime \prime \prime \prime} \rho .
$$

Since every symbol is invariant for a change of parameters, this is a covariant.

We see at once that for any six directions we have a similar form

$$
G_{6}=A_{0} \cdot d_{1} \rho d_{2} \rho d_{3} \rho A_{3} \cdot \Phi d_{4} \rho \Phi d_{5} \rho \Phi d_{6} \rho,
$$

and there will be higher forms as high as $G_{2 n}$. These are all covariants, and are generalizations of the Christoffel quadrilinear covariant $G_{4}$. 
10. From these we derive covariant quotients, which give us in the first instance the Riemann curvature for the plane of $d_{1} \rho, d_{2} \rho$, and by generalizations, Riemann curvatures for the regions of $d_{1} \rho, d_{2} \rho, \cdots$. These are

$$
K_{m}\left(d_{1} \cdots d_{m}\right)=\frac{A_{0} \cdot A_{m} \cdot d_{1} \rho d_{2} \rho \cdots d_{m} \rho A_{m} \Phi d_{1} \rho \Phi d_{2} \rho \cdots \Phi d_{m} \rho}{A_{0} \cdot A_{m} \cdot d_{1} \rho d_{2} \rho \cdots d_{m} \rho A_{m}} \cdot \frac{d_{1} \rho d_{2} \rho \cdots d_{m} \rho}{.}
$$

When we arrive at the covariant of order $K_{n}$ it is evidently the single coefficient of the Christoffel form for the same order. This number is called the Kronecker-Gaussian curvature of the space.

11. If we construct the second differential of $\rho, d^{2} \rho$, we have two parts to it, namely, that part which has components in the region, and the part which is orthogonal to the region. Since we have identically

it follows that

$$
A_{n+1} \cdot U \nu d \rho=0,
$$

But

$$
A_{n+1} \cdot d U \nu d \rho=-A_{n+1} \cdot U \nu d^{2} \rho
$$

$d^{2} \rho=\sum \rho_{i} A_{0} \cdot \rho^{i} d^{2} \rho+A_{1} \cdot U \nu A_{n+1} \cdot U \nu d^{2} \rho$

$$
=\sum \rho_{i} A_{0} \cdot \rho^{i} d^{2} \rho-A_{1} \cdot U \nu A_{n+1} \cdot d U \nu d \rho .
$$

We set now

$$
\xi=A_{1} \cdot U_{\nu} A_{n+1} \cdot d U_{\nu} d \rho .
$$

It is evident that $A_{0} \cdot \rho_{i} \xi=0$ for $i=1, \cdots, n$, so that $\xi$ is not in the region. $\xi$ is called the vector second differential form since the coefficients of its expansion are scalar second differential forms.* We evidently have

$$
\xi=\sum A_{1} \cdot U_{\nu} A_{n+1} \cdot\left(U_{\nu}\right)_{r} \rho_{s} d u_{r} d u_{s} .
$$

The coefficients $A_{1} \cdot U_{\nu} A_{n+1}\left(U_{\nu}\right)_{r} \rho_{s}$ are fundamental vectors of second order. They depend upon the parameters and are not covariants, but they furnish a number of covariants. We see that

Also

$$
A_{n+1} \cdot d U \nu d \rho=-A_{n+1} \cdot U \nu \xi \text {. }
$$

But in general

$$
A_{1} \cdot U \nu A_{n+1} \cdot d U \nu d \rho=-A_{1} \cdot U_{\nu} A_{n+1} \cdot U \nu \xi .
$$

$A_{1} \cdot A_{n} \alpha_{1} \alpha_{2} \cdots \alpha_{n} A_{n+1} \gamma \beta_{1} \beta_{2} \cdots \beta_{n}$

hence

$$
=(-)^{n} \gamma A_{0} \cdot A_{n} \cdot \alpha A_{n} \beta+(-)^{n} A_{1} \cdot\left(A_{n-1} \cdot \gamma A_{n} \cdot \alpha\right) \cdot A_{n} \beta \text {, }
$$

$$
\begin{aligned}
A_{1} \cdot U_{\nu} A_{n+1} \cdot d U \nu d \rho & =d \rho A_{0} \cdot U \nu d U \nu+A_{1} \cdot\left(A_{n-1} \cdot d \rho A_{n} \cdot U_{\nu}\right) A_{n} \cdot d U_{\nu} \\
& =A_{1} \cdot\left(A_{n-1} \cdot d \rho A_{n} \cdot U \nu\right) A_{n} \cdot d U \nu,
\end{aligned}
$$

*Cf. Wilson and Moore, Proceedings of the American Academy of Arts and Sciences, vol. 52 (1916), pp. 270-368. 
and also

$$
A_{1} \cdot U \nu A_{n+1} \cdot U \nu \xi=\xi+A_{1} \cdot\left(A_{n-1} \cdot \xi U_{\nu}\right) A_{n} \cdot U_{\nu} .
$$

But $\xi$ is orthogonal to all vectors $\rho$, hence $A_{n-1} \cdot \xi U_{\nu}=0$. Hence

$$
\xi=A_{1} \cdot\left(A_{n-1} d \rho U \nu\right) A_{n} \cdot d U_{\nu} .
$$

If we go back to the theory of curves we see that if we write the differential arc in the usual form $d \rho=d s \cdot \alpha,{ }^{*}$ then $d^{2} \rho=d^{2} s \alpha+d s d \alpha$ and as $A_{0} \alpha d \alpha=0$ it follows that the part of $d^{2} \rho$ outside the region consists of part of the term containing $d \alpha$. If $d \alpha=c \beta d s, \xi=A_{1} \cdot A_{n} \cdot U \nu A_{n+1} \cdot U \nu \beta \cdot c \cdot d s^{2}$, which is the part of $\beta \cdot c \cdot d s^{2}$ orthogonal to every vector $\rho_{1}, \cdots, \rho_{n}$.

12. It is evident that the first differential quadratic and whatever is derivable from it of a covariantive or invariantive character will be true of a region which is flexible but inextensible. That is to say, bending the region would have no effect upon the first fundamental form nor upon the quantities derived from it without differentiation. However the second fundamental vector form does not possess this character and demands a rigid space. This is due to the fact that $d^{2} \rho$ contains a part which is not in the region itself. For instance if we are dealing with a curve in three or more dimensions, the first fundamental form is merely $d s^{2}$, and as long as we do not consider $d^{2} \rho$ we merely work in the line itself which for all such purposes might as well be straight. If however we consider $d^{2} \rho=d^{2} s \cdot \alpha+d s \cdot d \alpha$ where $\alpha$ is the unit tangent (or better the hypernumber belonging to that particular tangent), then we must either abandon the line or consider $d \alpha=0$ which makes the line really straight.

It is to be noticed that the expressions (Christoffel symbols) $A_{0} \cdot \rho_{r s} \rho_{t}$ can be found from the region itself, since

$$
2 A_{0} \rho_{r s} \rho_{t}=\left(A_{0} \rho_{r} \rho_{t}\right)_{s}+\left(A_{0} \rho_{s} \rho_{t}\right)_{r}-\left(A_{0} \rho_{r} \rho_{s}\right)_{t},
$$

all of which could be computed in a practical case from measurements inside the region. From these we can compute the expressions $A_{0} \cdot \rho_{r s} \rho^{t}$. The Riemann symbols are also computable in the same manner, since

$$
\begin{aligned}
(r t, s u)= & \left(A_{0} \cdot \rho_{r s} \rho_{t}\right)_{u}-\left(A_{0} \cdot \rho_{r u} \rho_{t}\right)_{s} \\
& +\sum\left[A_{0} \cdot \rho^{h} \rho^{k}\left(A_{0} \cdot \rho_{r u} \rho_{h} A_{0} \cdot \rho_{s t} \rho_{k}-A_{0} \cdot \rho_{r s} \rho_{h} A_{0} \cdot \rho_{t u} \rho_{k}\right)\right]
\end{aligned}
$$

Covariants built out of these forms can likewise be computed directly in the space, and would be independent of any particular directions such as $r, s, t, u$.

13. If $\alpha=U d \rho$, then $d \alpha$ has two components, one in the region, and one exterior to the region. The latter is $\xi / d s$. The former is called the geodesic vector curvature of the curve of which $\alpha$ is the tangent. If it vanishes the curve is called a geodesic for the region. This may also be found as a curve of

* Where $\alpha$ is the unit tangent vector. 
minimal length. For let

then

$$
s=\int \sqrt{A_{0} \cdot d \rho d \rho}
$$

$$
\delta s=\int \delta \sqrt{A_{0} d \rho d \rho}=\int A_{0} d \delta \rho d \rho / d s=\int A_{0} d \delta \rho \alpha .
$$

Integrating by parts, the first part being then exact and vanishing for an extremal, we have as the condition of a minimum (or maximum) $A_{0} \delta \rho d \alpha=0$ for every $\delta \rho$ in the space. That is to say, we have $d \alpha$ wholly exterior to the space. We might also write this fact in the form

$$
A_{n-1} d \alpha A_{n} U_{\nu}=0 \text {. }
$$

It is understood here of course that the $d \alpha$ is for a displacement along the curve.

14. The covariant $G_{4}$ can be written

$$
G_{4}=A_{0} \cdot A_{2} \cdot d_{1} \rho d_{2} \rho A_{2} \cdot d_{3} U \nu d_{4} U \nu
$$

and is thus dependent upon the four directions of differentiation. Now we have

$$
d U \nu=d(\nu / I \nu)=d \nu / I \nu-U \nu A_{0} U \nu d \nu / I \nu
$$

Hence as $A_{2} A_{n} \alpha_{1} \cdots \alpha_{n} A_{n} \alpha_{1} \cdots \alpha_{n}=0,{ }^{*}$ and therefore $A_{2} \cdot U \nu U \nu=0$, $G_{4}=A_{0} \cdot A_{2} \cdot d_{1} \rho d_{2} \rho\left[A_{2} \cdot d_{3} \nu d_{4} \nu\right.$

$$
\left.-A_{2} \cdot U \nu\left(d_{3} \nu A_{0} \cdot d_{4} \nu-d_{4} \nu \cdot A_{0} \cdot d_{3} \nu\right) U \nu\right] / A_{0} \cdot \nu \nu \text {. }
$$

If we let four tangents to the space, that is, four unit directions, be $\alpha_{1}, \alpha_{2}, \alpha_{3}, \alpha_{4}$, we have

$$
K_{4}=A_{0} \cdot A_{2} \cdot \alpha_{1} \alpha_{2} A_{2} \cdot \Phi \alpha_{3} \Phi \alpha_{4} / A_{0} \cdot A_{2} \cdot \alpha_{1} \alpha_{2} A_{2} \cdot \alpha_{3} \alpha_{4} \cdot
$$

The Riemann symbol ( $r s, t u$ ) may be computed from observations in the space as follows:

We find first

$$
\left(A_{0} \cdot \rho_{r t} \rho_{s}\right)_{u}-\left(A_{0} \cdot \rho_{r u} \rho_{s}\right)_{t} .
$$

Then we find in the region $A_{0} \rho_{r t}^{\prime} \rho_{s u}^{\prime}-A_{0} \cdot \rho_{r u}^{\prime} \rho_{s t}^{\prime}$ which is subtracted from the other value $(\S 12)$. The difference is $(r s, t u)$. The accents on $\rho$ indicate that we are measuring so much of $\rho_{r t}$, etc., as we observe in the region in question. In the first expression these accents are not needed, as the form shows that the other parts of the second derivatives drop out anyhow. If we multiply by $d u_{u}$ and $d u_{t}$ and sum for all values of the differentials we have

$$
\begin{gathered}
d_{1} A_{0} \cdot d_{2} \rho_{r} \rho_{s}-d_{2} A_{0} \cdot d_{1} \rho_{r} \rho_{s}-A_{0} \cdot d_{2} \rho_{r}^{\prime} d_{1} \rho_{s}^{\prime}+A_{0} \cdot d_{1} \rho_{r}^{\prime} d_{2} \rho_{s}^{\prime} . \\
{ }^{*} A_{2} A_{n} \alpha_{1} \cdots \alpha_{n} A_{n} \alpha_{1} \cdots \alpha_{n}=\Sigma A_{0} A_{n-1} \alpha_{1} \cdots \alpha_{n} A_{n-1} \alpha_{1} \dddot{j} \alpha_{n} A_{2} \alpha_{i} \alpha_{i} \text {, which will } \\
\text { contain }\left(A_{2} \alpha_{i} \alpha_{i}+A_{2} \alpha_{i} \alpha_{i}\right) A_{0} A_{n-1} \alpha_{1} \cdots \alpha_{n} A_{n-1} \alpha_{1} \cdots \alpha_{n} \text {, and thus all terms cancel. }
\end{gathered}
$$


Multiply again by $d u_{\mathrm{s}} d u_{\mathrm{r}}$ and sum again, and we have

$$
\begin{aligned}
G_{4}=d_{1} A_{0} \cdot d_{2} d_{3} \rho d_{4} \rho-d_{2} A_{0} & \cdot d_{1} d_{3} \rho d_{4} \rho \\
& -A_{0} \cdot d_{3} d_{2} \rho^{\prime} d_{1} d_{4} \rho^{\prime}+A_{0} \cdot d_{1} d_{3} \rho^{\prime} d_{2} d_{4} \rho^{\prime} .
\end{aligned}
$$

This is the best form for this quadrilinear covariant, since by choosing the directions of the four differentials properly we may obtain any one of the Riemann forms.

15. The Riemann-Christoffel tensor $G_{4}^{\prime \prime}$ can be written in the form

$$
\left(A_{0} \cdot A_{2} \cdot \alpha \beta A_{2} \cdot \nabla_{1} \nabla_{2} A_{0} \cdot \gamma \delta\right)^{\prime \prime} \text {, }
$$

where $\nabla_{1}$ acts on $\gamma$ only, $\nabla_{2}$ on $\delta$ only, $\alpha, \beta$ are purely arbitrary, the double accent means that only components exterior to the space are concerned. $\alpha, \beta, \gamma, \delta$ are unit vectors. If this is to vanish for any $\alpha, \beta$, then

$$
\left(A_{2} \cdot \nabla_{1} \nabla_{2} A_{0} \cdot \gamma \delta\right)^{\prime \prime}=0,
$$

and this may be written

$$
\left(A_{2} \cdot \nabla \phi_{\gamma}^{\prime} \delta\right)^{\prime \prime}=0,
$$

where $\phi_{\gamma}$ is the derivative dyad of $\gamma$, or $\phi_{\gamma} \zeta d t=d_{\zeta} \gamma$, the differential of $\gamma$ in the $\zeta$ direction. In other terms $\phi_{\gamma}=A_{0} \cdot() \nabla \cdot \gamma$ and $\phi_{\gamma}^{\prime}=\nabla A_{0} \cdot \gamma()$.

If this vanishes for every $\delta$ we must have $\left(A_{2} \cdot \nabla \gamma\right)^{\prime \prime}=0$. This can vanish for every $\gamma$ only when all the Riemann symbols vanish, which interprets the meaning of the vanishing of all these symbols.

16. The Einstein gravitation equations translated into our notation are

$$
\begin{aligned}
G_{i j}=-\left(\sum A_{0} \cdot \rho_{i j}^{\prime} \rho^{k}\right)_{k}-\sum A_{0} \cdot \rho_{i k}^{\prime}\left(\rho^{k}\right)_{j} & \\
& \quad+\left(A_{0} \cdot \nabla \rho_{i}\right)_{j}+\sum A_{0} \cdot \rho_{i j}^{\prime}\left(\rho^{k}\right)_{k} \\
= & -A_{0} \nabla \rho_{i j}^{\prime}-\sum A_{0} \cdot \rho_{i j}^{\prime}\left(\rho^{k}\right)_{k}-\sum A_{0} \cdot \rho_{i k}^{\prime}\left(\rho^{k}\right)_{j} \\
& \quad+\left(A_{0} \cdot \nabla \rho_{i}\right)_{j}+\sum A_{0} \cdot \rho_{i j}^{\prime}\left(\rho^{k}\right)_{k} \\
= & -A_{0} \cdot \nabla \rho_{i j}^{\prime}-\sum A_{0} \cdot \rho_{i k}^{\prime}\left(\rho^{k}\right)_{j}+\left(\sum A_{0} \rho^{k} \rho_{i k}\right)_{j} \\
= & -A_{0} \cdot \nabla \rho_{i j}^{\prime}-\sum A_{0} \cdot \rho_{i k}^{\prime}\left(\rho^{k}\right)_{j}+\sum A_{0} \cdot \rho_{i k}^{\prime}\left(\rho^{k}\right)_{j}+\sum A_{0} \cdot \rho^{k} \rho_{i j k} \\
= & -A_{0} \cdot \nabla \rho_{i j}^{\prime}+A_{0} \cdot \nabla \rho_{i j}=\left(A_{0} \cdot \nabla \rho_{i j}\right)^{\prime \prime}=0 \text { for all } i, j:
\end{aligned}
$$

Therefore the significance is easily seen to be that the convergence of the exterior parts of the second differentials is zero. That is, if $\alpha$ is a unit tangent, $A_{0} \cdot \nabla d \alpha^{\prime \prime}=0$. Hence the sum of the normal curvatures of $n$ orthogonal geodesics at the point is zero. The Einstein curvature is

$$
\sum g^{i j} G_{i j}=\sum A_{0} \cdot \rho^{t} \nabla^{2} \rho_{t}^{\prime \prime}=\sum_{r s} A_{0} \cdot \rho^{r} \rho^{s} A_{0} \cdot \rho^{t} \rho_{t r s} .
$$

The Riemann-Christoffel covariant is written also without the accents (since components in the space lead to terms that cancel)

$$
A_{0} \cdot A_{2} \cdot \alpha \beta A_{2} \cdot \nabla_{1} \nabla_{2} A_{0} \cdot \gamma \delta,
$$


and if we substitute for $\gamma$ the adjunct of $\beta$, and sum for $n$ linearly independent values $\beta_{i}$ with $\beta^{i}$, we have the Einstein covariant in the form

$$
G_{\alpha \delta}=\sum A_{0} A_{2} \alpha \beta_{i} A_{2} \nabla_{1} \nabla_{2} A_{0} \beta^{i} \delta=0 \text { for all } \alpha, \delta .
$$

This gives $\sum A_{0} A_{2} \alpha \beta_{i} A_{2} \nabla \phi_{\delta}^{\prime} \beta^{i}$ where $\nabla$ operates only on the $\beta^{i}$. This takes the form

$$
\sum\left(A_{0} \cdot \phi_{\delta} \alpha \beta^{i} A_{0} \beta_{i} \nabla-A_{0} \alpha \nabla A_{0} \beta^{i} \phi_{\delta} \beta_{i}\right) ;
$$

which is another form of the potential.

If we start from the forms $\left(A_{0} \cdot \rho_{t r} \rho^{8}\right)_{u}^{\prime \prime}-\left(A_{0} \cdot \rho_{r u} \rho^{8}\right)_{t}^{\prime \prime}$ and, making $s=u$, sum, we have

$$
G_{r t}=A_{0} \cdot \nabla \rho_{r t}+\sum A_{0} \rho_{r t}\left(\rho^{s}\right)_{s}=\left(A_{0} \nabla \rho_{r}\right)_{t}=A_{0} \cdot \nabla \rho_{r t}^{\prime \prime}
$$

as before.

The simplest way to get the second derivatives is to choose paths in the region on the geodesics. There is then no normal in the region. If then the convergence in the region of these geodesic normals vanishes, the space is of the gravitational character.

If the Riemann-Christoffel covariants vanish for an ordinary space we know it is a plane, and a surface nabla of the normals (which would run out into three-dimensional space) all vanish. To be an Einstein space of two dimensions it would be necessary for the convergence in the space of the geodetic normals to vanish. For a sphere, for instance, the normals all pass through the center, their differentials have any direction on the surface, and would not necessarily give a convergence equal to zero. A sphere is therefore not a possible Einstein surface.

\section{Tensors}

17. As commonly used the term tensor means merely a multiplex of coefficients obtained when we substitute in a lineo-linear function of $m$ variables the various vectors chosen $m$ at a time from the set $\rho_{1}, \cdots, \rho_{n}$. We shall use $\Xi$ to indicate a tensor, which from our point of view is an operator. To indicate the operands we will insert $m$ parentheses after $\Xi$. A tensor is an extension of the term dyad, triad, etc., used by Gibbs. We will write then a tensor of order 1 as $\boldsymbol{\Xi}()$, one of order 2 as $\boldsymbol{\Xi}()()$, etc. The first fundamental form is a tensor since it has the form $A_{0} \cdot()()$. From it we derive the set of quantities $A_{0} \cdot \rho_{r} \rho_{s}$. It is clear that all our $A$ symbols, $A_{m}, \cdots$ furnish tensors. A tensor is a covariant tensor when it reduces to a form independent of $\rho_{1}, \cdots, \rho_{n}$. All the symbols $A$ and their combinations furnish tensors that are covariant.

A tensor is a derivative tensor, and of order higher by unity than another, when it is formed from the other by the process

$$
\Xi()() \cdots() A_{0} \cdot \nabla() \text {. }
$$


For instance from the tensor $\xi$ (a covariant, as $\rho_{1}, \cdots, \rho_{n}$ are not in evidence) we derive $\xi A_{0} \nabla()$ where $\nabla$ operates only on $\xi$. If $\xi=\nabla X$ then we have $\nabla X A_{0} \nabla()$. If $\xi$ is a differential operator, as $A_{0} \cdot() \nabla \cdot \sigma$, then we derive

$$
A_{0} \cdot() \nabla \cdot \sigma \cdot A_{0} \cdot \nabla() \text {. }
$$

If we indicate direction by a subscript as $\sigma_{r}$, then the derived tensor demands that $\sigma_{r s}=\sigma_{s r}$. This condition is necessary. It is further a sufficient condition that $\Xi$ be a derived or differential tensor.

All differentials or derivatives taken in this manner without reference to the parameters $u$ are called covariant differentials. The so-called contravariant systems would be found by using the system $\rho^{i}$.

18. To make translations from the ordinary notations we have to notice that the covariant tensor $A_{x y}$ means the form $\sum A_{x y} A_{0} \cdot \rho^{x}() A_{0} \cdot \rho^{\nu}()$, the contravariant tensor $A^{x y}$ means $\sum A^{x y} A_{0} \cdot \rho_{x}() A_{0} \cdot \rho_{y}()$, and the mixed tensor $A_{x}^{y}$ means $\sum A_{x}^{y} A_{0} \cdot \rho^{x}() A_{0} \cdot \rho_{y}()$.

The so-called product of two tensors of orders $a, b$ is a tensor of order $a+b$. For instance the product $A_{u v} B_{s}^{r}$ means

$$
\sum A_{u v} B_{s}^{r} A_{0} \cdot \rho^{u}() A_{0} \cdot \rho^{v}() A_{0} \cdot \rho^{s}() A_{0} \cdot \rho_{s}() \text {. }
$$

In expressions where "dummy" indices appear, that is to say, duplicated indices, the expressions are summed as to the operational terms indicated, for instance, $A_{u r s}^{z}$ means $\sum_{s} A_{u r s}^{s} A_{0} \cdot \rho^{u}() A_{0} \cdot \rho^{r}$ () $A_{0} \cdot \rho^{s}$ ( ) $A_{0} \cdot \rho_{s}$ ( ).

We will translate some of the current notations into these forms:

$$
\begin{aligned}
a_{u v}, w & =[u v, w]=A_{0} \cdot \rho_{u v} \rho_{w}, & \{u v, w\} & =A_{0} \cdot \rho_{u v} \rho^{w}, \\
A^{i} & =\sum A^{i} A_{0} \cdot \rho^{i}(), & \left(A^{i}\right)_{j} & =\sum A_{1}^{i} A_{0} \cdot \rho^{i}() A_{0} \cdot \nabla_{1}(),
\end{aligned}
$$

where the subscript 1 shows the operand of $\nabla$. This is covariantive differentiation.

With these indications it is easy to reduce any expression into the vector forms, and then to reduce the vector forms to compact expressions which from their forms alone show that they are covariantive.

\section{Curves}

19. Let $\alpha$ be the unit tangent to a curve (that is, a function of one parameter s), which gives $A_{0} \cdot \alpha \alpha=1$. We have therefore at once

$$
A_{0} \cdot \alpha d \alpha=0 \text {. }
$$

Hence $d \alpha$ is orthogonal to $\alpha$. Let $\beta$ be a unit vector so that

$$
d \alpha=c_{1}^{\prime} \beta d s .
$$

$c_{1}^{\prime}$ is called the curvature and $\beta$ the principal normal. Usually part of $\beta$ is 
in the space of the curve, part not in the space. For instance, a curve on a sphere may have its principal normal lying in the embedding three-dimensional space. We may also write, then,

$$
d \alpha=\left(g_{1} \lambda+n_{1} \nu\right) d s,
$$

where now $g_{1}$ is the geodesic curvature, $n_{1}$ is the normal curvature, $\lambda$ is a unit normal in the space, and $\nu$ is a unit normal out of the space.

Since $A_{0} \cdot \alpha \beta=0$, differentiating again, $A_{0} \cdot \alpha d \beta=-A_{0} \cdot \beta d \alpha=-c_{1}^{\prime}$. Hence we have for $d \beta$ (the $d \rho$ is still along the curve)

$$
d \beta=-c_{1}^{\prime} \alpha+c_{1}^{\prime \prime} \gamma,
$$

where $\gamma$ is called the binormal, and $c_{1}^{\prime \prime}$ is the second curvature. Since the first term gives the entire projection on $\alpha$ we must have $A_{0} \cdot \alpha \gamma=0$, and since $A_{0} \cdot \beta d \beta=0$, we must have also $A_{0} \cdot \beta \gamma=0$. Continuing we find the trinormal, etc., and the third, etc., curvatures, giving the generalized Frenet equations:

$$
\begin{aligned}
& d \alpha=c_{1}^{\prime} \beta d s, \\
& d \beta=\left(c_{1}^{\prime \prime} \gamma-c_{1}^{\prime} \alpha\right) d s, \\
& d \gamma=\left(c_{1}^{\prime \prime \prime} \delta-c_{1}^{\prime \prime} \beta\right) d s, \\
& \cdot \cdot \cdot \cdot \cdot \cdot \cdot \cdot \\
& d \mu=-c_{1}^{(m)} \kappa d s .
\end{aligned}
$$

When $c_{1}^{\prime}=0$ the other curvatures become indefinite but are taken as zero by convention. If $c_{1}^{\prime} \neq 0$ and $c_{1}^{\prime \prime}=0$, from the equations above

$$
A_{3} \cdot d \rho d^{2} \rho d^{3} \rho=0 .
$$

The condition is necessary and sufficient. Hence, differentiating,

$$
A_{3} \cdot d \rho d^{2} \rho d^{4} \rho=0=A_{3} \cdot d \rho d^{2} \rho d^{5} \rho,
$$

and all higher curvatures may be taken as zero.

20. A congruence of curves is defined by the equation

$$
A_{2} \cdot \lambda d \rho=0,
$$

where $\lambda$ is a given unit vector in the region.* ${ }^{*}$ Let us consider $n$ independent congruences, one curve of each congruence through any one point, and at the point let all the congruences be mutually orthogonal. To indicate the orthogonality we shall use $\alpha$ instead of $\lambda$. Then

$$
A_{0} \cdot \alpha_{i} \alpha_{h}=\delta_{i h},
$$

and using $\phi_{\alpha_{i}}$ to indicate $A_{0} \cdot() \nabla \cdot \alpha_{i}$, the operator that gives the differential

* The notation of Ricci is as follows in vector forms: $\lambda_{i / r}=A_{0} \cdot \lambda_{i} U_{\rho_{r}}, \lambda_{h}^{(r)}=A_{0} \cdot \lambda_{h} \rho^{r}$, where $i$ and $h$ define different congruences; $\lambda_{i / r s}=A_{0} \cdot \rho_{r}\left(\lambda_{i}\right)_{e}, \gamma_{i j k}=A_{0} \cdot \lambda_{j}\left(\lambda_{i}\right)_{k}$. 
of $\alpha_{i}$ in the direction of the unit operand, we have

$$
A_{0} \cdot \alpha_{i} \phi_{\alpha_{j}} \alpha_{k}=-A_{0} \cdot \alpha_{j} \phi_{\alpha_{i}} \alpha_{k} \text { for all } i \neq j \neq k \text {. }
$$

Such an expression is called a coefficient of rotation for a reason that will appear. There are $\frac{1}{2} n^{2}(n-1)$ such expressions. We have also

$$
A_{0} \cdot \alpha_{h} \phi_{\alpha_{h}} \alpha_{l}=0 \text { if } h \neq l \text {. }
$$

We may expand $A_{0} \cdot \rho_{r} \phi_{\alpha_{h}} \rho_{s}$ in terms of $\sum A_{0} \cdot \alpha_{i} \rho_{r} A_{0} \cdot \alpha_{j} \rho_{s} A_{0} \cdot \alpha_{i} \phi_{\alpha_{h}} \alpha_{j}$. This is the Ricci formula

$$
\lambda_{h / r s}=\sum \gamma_{h i j} \lambda_{i / r} \wedge_{j / s},
$$

but such expansion is not needed in the vector forms.

21. Let

$$
\theta=\frac{1}{2} \sum A_{2} \cdot \alpha_{i} \phi_{\alpha_{i}}()
$$

be an operator which converts vectors of grade unity into vectors of grade 2 . Of course it is a very special operator of this kind. The coefficient of rotation

$$
\gamma_{i j k}=A_{0} \cdot \alpha_{i} \alpha_{j} A_{2} \cdot \theta \alpha_{k} .
$$

We see that $\theta d \rho=\frac{1}{2} \sum A_{2} \cdot \alpha_{i} d \alpha_{i}$ gives what may be called the instantaneous rotation for a displacement $d \rho$ about a plane. That is to say, the plexus of congruences rotates about this plane through an infinitesimal amount given by the intensity of the 2-vector. (Rotation about a plane or other spread is possible in hyperspace.) We can look at the situation in this way: let

$$
\tau=A_{1} \cdot \sigma \theta \eta=-\sum \phi_{\alpha_{j}} \eta A_{0} \cdot \alpha_{j} \sigma
$$

and $A_{0} \cdot \alpha_{k} \tau=A_{0} \cdot \sigma \phi_{\alpha_{k}} \eta=A_{0} \cdot \sigma d \alpha_{k} / d t, A_{0} \cdot \sigma \tau=0$. The coefficients of rotation are the components in the directions of the elementary bivectors $A_{2} \cdot \alpha_{i} \alpha_{j}$ of the bivector $\theta \alpha_{k}$.

We can also express $\theta$ a little differently in a form useful in the study of n-tuply orthogonal congruences.*

Let

$$
\zeta=\sum A_{2} \cdot \nabla^{\prime} \alpha_{i}^{\prime} A_{0} \cdot \alpha_{i}()=-\sum A_{2} \cdot \nabla^{\prime} \alpha_{i} A_{0} \cdot \alpha_{i}^{\prime}(),
$$

the accents showing the operand of $\nabla$. Then

$$
\theta-\zeta=\frac{1}{2} A_{2} \text { () } \sum A_{3} \cdot \alpha_{i} \alpha_{i}^{\prime} \nabla^{\prime}=-\frac{1}{2} A_{2} \cdot() \sum A_{3} \alpha_{i} \nabla \alpha_{i} .
$$

The expression $A_{2} \cdot \nabla \alpha_{i}$ is of course the curl of $\alpha_{i}$.

22. If $f(\rho)=0$ is a spread of order $n-1$, the normals are $\nabla f$ and form a normal congruence. Since $f_{h k}=f_{k h}$, we have

$$
A_{0} \cdot \alpha_{h} \nabla A_{0} \cdot \alpha_{k} \nabla \cdot f-A_{0} \cdot \alpha_{k} \nabla A_{0} \cdot \alpha_{h} \nabla \cdot f=0 .
$$

* See Shaw, Triply orthogonal congruences, these Transaction s, vol. 21 (1920), p. 391. The present articles contain the results of a paper read December 31, 1915, before the Chicago section, On orthogonal congruences in space of three and more dimensions. 
As $\nabla$ operates upon following $\alpha$ 's we have from this

$$
A_{0} \cdot \nabla f\left(\phi_{\alpha_{h}} \alpha_{k}-\phi_{\alpha_{k}} \alpha_{h}\right)=0 .
$$

This might be written

$$
\sum\left(\gamma_{i k h}-\gamma_{i h k}\right) A_{0} \cdot \alpha_{i} \nabla f=0 .
$$

Let the unit part of $\nabla f$ be $\alpha_{n}$; then the equation gives

$$
A_{0} \cdot\left(\alpha_{n} \phi_{\alpha_{h}} \alpha_{k}-\alpha_{n} \phi_{\alpha_{k}} \alpha_{h}\right)=0 \text {. }
$$

That is, for the entire set of curves in the spread and normal to it,

$$
\gamma_{n k h}=\gamma_{n h k} \quad(h, k=1,2, \cdots, n-1) .
$$

The equation also states that $\phi_{\alpha_{k}}$ is self-transverse (symmetric) so far as the spread is concerned. The condition is also sufficient. For when it is satisfied $A_{0} \cdot A_{2} \cdot \alpha_{h} \alpha_{k} A_{2} \cdot \nabla \alpha_{0}=0$ for all $h, k=1, \cdots, n-1$. But

hence we have

$$
A_{2} \nabla \alpha_{n}=\sum x_{j} A_{2} \alpha_{n} \alpha_{j},
$$

$$
A_{3} \cdot \alpha_{n} \nabla \alpha_{n}=0 \text {, }
$$

whence $t A_{2} \cdot \nabla \alpha_{n}=A_{2} \xi \alpha_{n}$ for any $\xi$. Choose $\xi=-\nabla t$ and we have

$$
A_{2} \nabla\left(t \alpha_{n}\right)=0, \quad t \alpha_{n}=\nabla f \text {. }
$$

From this we have also $A_{2} \cdot \nabla \log t \alpha_{n}=-A_{2} \cdot \nabla \alpha_{n}=A_{2} \cdot \alpha_{n} \pi$, where $\pi$ is in the spread and is indeed $\phi_{\alpha_{n}} \alpha_{n}$.

23. Let us suppose now that $\alpha_{n}$ is the principal normal of a system of curves through the point, that is, $\alpha_{i}$ is the unit tangent, $d \alpha_{i} / d s$ for $d \rho$ along the curve is $c_{i} \alpha_{n}$, where $i=1, \cdots, n-1$. That is,

Hence

$$
\phi_{\alpha_{i}} \alpha_{i}=c_{i} \alpha_{n} \text { for all } i=1, \cdots, n-1 \text {. }
$$

$$
\begin{array}{r}
A_{2} \cdot \alpha_{n} \phi_{\alpha_{i}} \alpha_{i}=0, \quad A_{0} \cdot \alpha_{j} \phi_{\alpha_{i}} \alpha_{i}=-A_{0} \cdot \alpha_{i} \phi_{\alpha_{j}} \alpha_{i}=0 \\
(i, j=1, \cdots, n-1) .
\end{array}
$$

This may also be written to parallel the treatment in three variables referred to above:

$$
\begin{aligned}
A_{1} \cdot \alpha_{i} \Theta \alpha_{i} & =c_{i} \alpha_{n}, \\
A_{0} \cdot \alpha_{n} \phi_{\alpha_{i}} \alpha_{i} & =c_{i} .
\end{aligned}
$$

If the curves all lie in a spread as in the preceding article,

$$
\begin{aligned}
\nabla \log t & =\phi_{\alpha_{n}} \alpha_{n} \tau+x \alpha_{n}, \\
A_{0} \cdot \alpha_{n} \phi_{\alpha_{i}} \alpha_{j} & =A_{0} \cdot \alpha_{n} \phi_{\alpha_{j}} \alpha_{i} .
\end{aligned}
$$

In the general case of all the curves merely such that $\alpha_{n}$ is their common principal normal, the sum of the curvatures is

$$
A_{0} \cdot \alpha_{n} \sum \phi_{\alpha_{i}} \alpha_{i} \quad(i=1, \cdots, n) .
$$


which reduces to

$$
-\sum A_{0} \cdot \alpha_{n} A_{1} \cdot \alpha_{i} \theta \alpha_{i} \quad \text { or } \quad \sum-A_{0} \cdot \alpha_{i} \phi_{\alpha_{n}} \alpha_{i}=-A_{0} \cdot \nabla \alpha_{0} .
$$

This shows on the one hand that the sum of the curvatures of the normal curves is independent of the particular system chosen, if they are mutually orthogonal, but on the other hand it gives significance to the geometric convergence of $\alpha_{n}$. Dividing by $n-1$ would give the mean curvature of the system.

The second curvature of the curve with $\alpha_{i}$ as tangent is

$$
\phi_{\alpha_{n}} \alpha_{i}=c_{i}^{\prime \prime} \gamma_{i}-c_{i}^{\prime} \alpha_{i}
$$

Since $\alpha$ and $\gamma$ are orthogonal,

and we may write

$$
A_{2} \cdot \alpha_{i} \phi_{\alpha_{n}} \alpha_{i}=c_{i}^{\prime \prime} \alpha_{i} \gamma_{i}
$$

$$
c^{\prime \prime} \text { is the intensity of } A_{2} \cdot \alpha_{i} \phi_{\alpha_{n}} \alpha_{i} \text {. }
$$

24. The spread of order $n-1$ is called isothermal when

$$
A_{0} \nabla \nabla f=0=\nabla^{2} f .
$$

The resulting equations are simple.

25. In case the curves in the region through the point in question are geodesic we have $\phi_{\alpha_{n}}, \alpha_{n}=0$ in the region, that is, for every $i$

That is,

$$
A_{0} \cdot \alpha_{i} \phi_{\alpha_{n}} \alpha_{n}=0 \text {. }
$$

$$
A_{0} \cdot \alpha_{n} \phi_{\alpha_{i}} \alpha_{n}=0
$$

for every $i$. This may also be written $A_{0} \cdot \alpha_{n} \alpha_{i} \theta \alpha_{n}=0$. This form enables us to state the condition in the form $A_{1} \cdot \alpha_{n} \theta \alpha_{n}=0$ in the region, and this vector must lie outside the region therefore. ${ }^{*}$

Further applications must be deferred.

* See page 213 .

UNIVERSITY OF ILLINOIS, URBANA, ILL. 